Acta Crystallographica Section A

Foundations and

Advances

ISSN 2053-2733

Received 13 October 2013

Accepted 11 March 2014

(C) 2014 International Union of Crystallography

\section{Unification and classification of two-dimensional crystalline patterns using orbifolds ${ }^{1}$}

\author{
S. T. Hyde, ${ }^{a, b *}$ S. J. Ramsden ${ }^{a}$ and V. Robins ${ }^{a}$
}

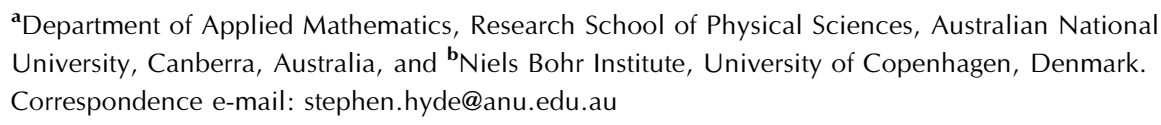

The concept of an orbifold is particularly suited to classification and enumeration of crystalline groups in the euclidean (flat) plane and its elliptic and hyperbolic counterparts. Using Conway's orbifold naming scheme, this article explicates conventional point, frieze and plane groups, and describes the advantages of the orbifold approach, which relies on simple rules for calculating the orbifold topology. The article proposes a simple taxonomy of orbifolds into seven classes, distinguished by their underlying topological connectedness, boundedness and orientability. Simpler 'crystallographic hyperbolic groups' are listed, namely groups that result from hyperbolic sponge-like sections through three-dimensional euclidean space related to all known genus-three triply periodic minimal surfaces (i.e. the $P, D$, Gyroid, $C L P$ and $H$ surfaces) as well as the genus-four $I-W P$ surface.

\section{Introduction}

Our prime aim here is to advocate and explore a comprehensive, unified schema to describe groups of isometries of the three two-dimensional spaces of constant intrinsic (Gaussian) curvature, namely the (flat) euclidean plane $\left(\mathbb{E}^{2}\right)$, the (elliptic) sphere $\left(\mathbb{S}^{2}\right)$ and the hyperbolic plane $\left(\mathbb{H}^{2}\right)$. Groups in $\mathbb{E}^{2}$ and $\mathbb{S}^{2}$ are better known to crystallographers as plane and point groups, respectively. Standard references of crystallography, such as the International Tables (Hahn, 1992), give detailed accounts of these subgroups of three-dimensional (euclidean) space groups. Conventional crystallography highlights the role of the translational unit cell, the infinite crystalline pattern modulo its translational symmetries. An alternative approach is possible with 'orbifolds', which contain rather a single asymmetric domain of the pattern (Conway \& Huson, 2002; Conway et al., 2008). The possibility of employing orbifolds in a crystallographic context has been explored previously (Johnson \& Burnett, 1996). It has also been discussed within a crystallographic context by the mathematicians Conway and Huson (Conway \& Huson, 2002), using Conway's orbifold nomenclature, that we adopt here (in contrast to Johnson's modified symbols, that appear to us unnecessary). Emil Molnár has also discussed the two-dimensional symmetries of 3-periodic minimal surfaces using Conway's orbifolds (Molnar, 2002); a concept discussed independently by us (Hyde \& Ramsden, 2003). This topological approach to crystallography affords a new way of thinking about symmetric

\footnotetext{
${ }^{1}$ This article forms part of a special issue dedicated to mathematical crystallography, which will be published as a virtual special issue of the journal in 2014.
}

patterns and, by implication, crystallography itself (Conway, 1992).

This approach is not new to geometry; it is however novel to most practising crystallographers. We therefore describe the salient features of two-dimensional orbifolds in some detail, revisiting the work of (principally) Conway and colleagues, and focus on aspects of relevance to crystallography. We then present a new taxonomy of orbifolds, developed in order to manage the abundant wealth of hyperbolic examples, with the definition of seven distinct orbifold classes. This taxonomy emerges clearly from the topological differences between orbifolds in distinct classes. Lastly, we introduce the notion of 'crystallographic' hyperbolic orbifolds, that are commensurate with crystalline patterns in conventional three-dimensional euclidean space $\left(\mathbb{E}^{3}\right)$, and present a comprehensive list of 'crystallographic' hyperbolic orbifolds, which can be realized in three-dimensional euclidean space via all simpler crystalline (i.e. 3-periodic) hyperbolic minimal surfaces $(P, D$, Gyroid, $I-W P, H$ and $C L P$ surfaces).

Analogues of the point and plane groups in the hyperbolic plane cannot be accommodated by simple extensions of the standard Hermann-Mauguin or Schoenflies names; in contrast the orbifold approach affords a useful universal naming scheme. Given that groups of isometries of the hyperbolic plane are overwhelmingly rich compared with plane and point groups, we recast point and plane groups within the orbifold mould to arrive at a single coherent schema for all three twodimensional spaces.

This proposed reconstruction of two-dimensional crystallography is motivated by advances in our understanding of structures in real materials that demand the inclusion of groups of isometries of $\mathbb{H}^{2}$ within the spectrum of low- 
dimensional crystallography. Until recently, it was widely accepted that two-dimensional hyperbolic geometry - while undoubtedly elegant - bore little connection with the real world. For example, an otherwise excellent mathematical text devoted to symmetric tessellations contains the following caveat applied to tessellations of $\mathbb{H}^{2}$ (Montesinos, 1987): 'It does not seem likely that examples of such tessellations will be found in Nature.' On the other hand, the need to generalize crystallography was foreseen already in the 1940s by Bernal as a consequence of the occurrence of helical and icosahedral symmetries in biological molecules (Bernal, 1966). More recently, Mackay and colleagues have argued that developments in materials science make that task more urgent than ever (Shevchenko et al., 2007). They point out that the atomic structures within nanoparticles may adopt structures whose extensions to global patterns are impossible in threedimensional euclidean space. They write: 'There is nothing bad that the idealized structure as a whole cannot be embedded into Euclidean space. Its fragments inherit partially the non-Euclidean symmetry which becomes hidden. Highly symmetrical idealizations should be chosen either in symmetrical spaces of positive or negative curvature, or in projective spaces, or more generally, in a certain fibre space.' In other words, Montesinos' claim need not preclude the exploration of non-euclidean patterns as 'approximants' of actual structures in our flat space. A stronger objection to his claim lies however in the wealth of materials, from the atomic to the polymolecular scale, whose structures can be described as tessellations of hyperbolic surfaces, particularly triply periodic minimal surfaces or 'TPMSs' (see, for examples, Hyde et al., 1997). The two-dimensional intrinsic geometries of patterns on TPMSs are characterized by groups of isometries of $\mathbb{H}^{2}$. The recognition of hyperbolic two-dimensional geometries in crystalline materials at many length scales surely demands extension of two-dimensional crystallography to accommodate the hyperbolic case.

In addition to the presence of hyperbolic surfaces in crystalline materials, the case for inclusion of hyperbolic symmetry groups within crystallography can be argued from a more fundamental perspective still, namely that of crystalline versus aperiodic patterns in our space. Alan Mackay, who pioneered the exploration of quasicrystalline patterns at a theoretical level, recognized the possibility of 'forbidden' (sevenfold) symmetries on surfaces of negative curvature (Mackay, 1986). Just whether and how those hyperbolic symmetries can be realized in flat 3-space is, in general, a complex issue. Evidently, crystalline patterns cannot be realized in $\mathbb{E}^{3}$ with sevenfold symmetry. Here we address a simpler issue, namely, which two-dimensional hyperbolic groups are commensurate with crystalline patterns in 3-space. A preliminary list of the most symmetric 'crystallographic two-dimensional hyperbolic groups' is presented.

The case for a new naming schema for point and plane groups is clearly one that cannot be made lightly. We are, however, convinced that such a step is overdue if hyperbolic symmetries are to be treated within the same framework as elliptic and planar cases. Given the overwhelming richness of

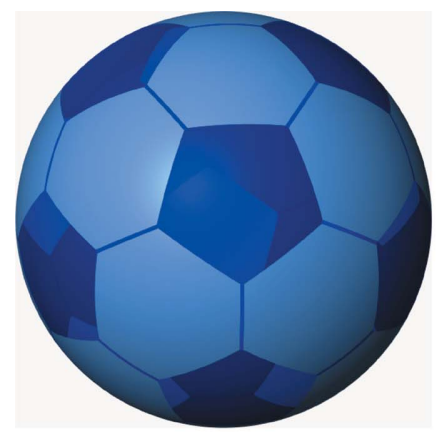

(a)

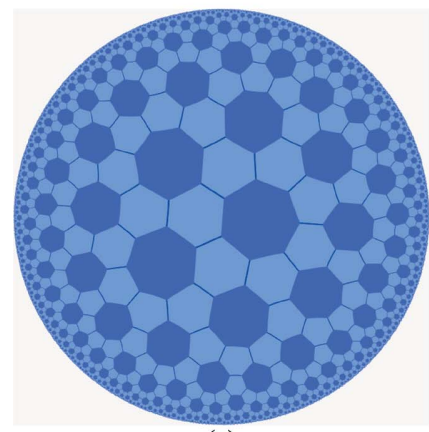

(c)

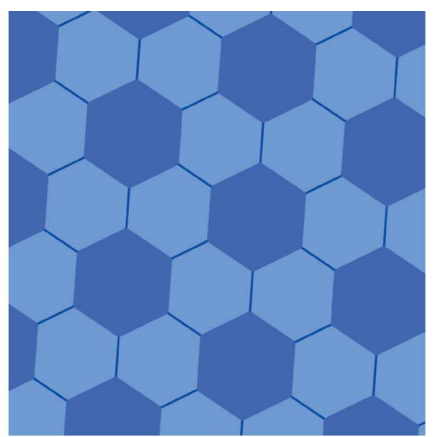

(b)

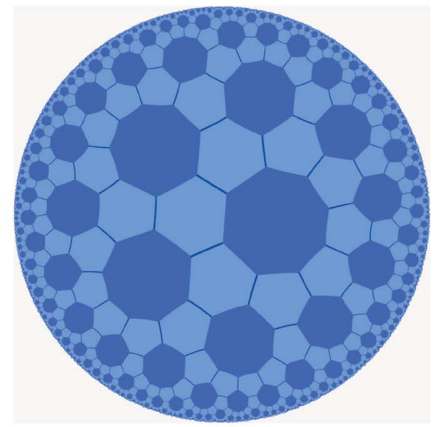

(d)
Figure 1

(a) The $\mathrm{C}_{60}$ net on the sphere $\mathbb{S}^{2}$, whose symmetries are those of the $\star 235$ orbifold. (b) The hcb net, with $\star 236$ orbifold. Hyperbolic mutations of the same net, drawn in the Poincaré disc model of $\mathbb{H}^{2}$, with orbifolds $(c) \star 237$ and $(d) \star 238$, respectively.

groups of isometries of (hyperbolic) $\mathbb{H}^{2}$ compared with the more familiar cases of (flat) $\mathbb{E}^{2}$ and (elliptic) $\mathbb{S}^{2}$, and the elegance and relative simplicity of the orbifold approach, it is useful to gather all three spaces within the common framework of orbifolds. That allows, for example, morphing of tessellations from one space to the others via 'symmetry mutation', already discussed within the crystallographic context by Huson (Huson, accessed 2013).

Consider, for example, the $s p^{2}$ bonding network in the fullerene, $\mathrm{C}_{60}$. The net has two-dimensional Schläfli symbol (5.6.6), corresponding to the polygonal faces coincident to each vertex. We can project the net onto $\mathbb{S}^{2}$, giving a symmetric pattern with identical vertices defined by a spherical triangular asymmetric domain, with 2, 3 and 5 mirror lines meeting at each vertex (Fig. 1a). This domain is the coxeter (kaleidosopic) orbifold $\star 235$, which defines a group of isometries of $\mathbb{S}^{2}$ (or equivalently, the $I_{h}$ point group). (The nomenclature is described in detail below.) If the same motif (edges and vertex per asymmetric unit) are embedded in a mutated domain with an additional mirror line to give the $\star 236$ orbifold, the resulting net is now planar (tiling $\mathbb{E}^{2}$ ) and is the familiar (6.6.6) graphene net (Fig. 1b). We can further mutate the pattern by adding additional mirror lines, forming a (6.6.7) net in $\mathbb{H}^{2}$, or more generally (6.6.k) nets from $\star 23 \mathrm{k}$ where $k$ is an integer $>6$ (Figs. $1 c$ and $d$ ).

In contrast to patterns on $\mathbb{S}^{2}$ or $\mathbb{E}^{2},(6.6 . k)$ nets in $\mathbb{H}^{2}$ cannot be embedded in euclidean 3 -space $\left(\mathbb{E}^{3}\right)$ via TPMSs without losing some of their isometries. This subtlety is due to the generic incommensurability between $\mathbb{H}^{2}$ and $\mathbb{E}^{3}$. While $\mathbb{S}^{2}$ and 


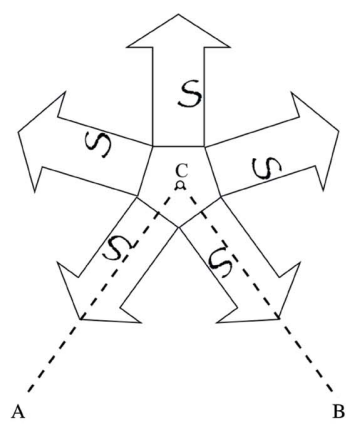

(a)

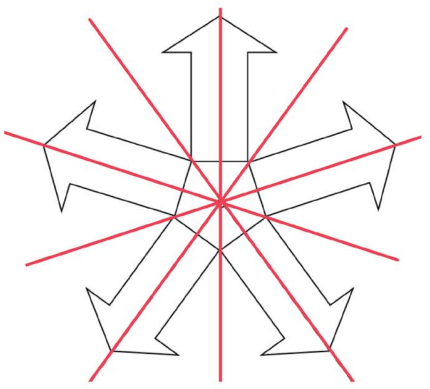

(c)

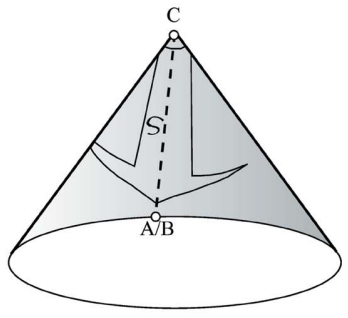

(b)

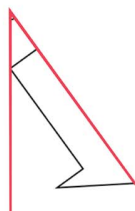

(d)
Figure 2

(a) Identifying all equivalent points on a rosette pattern with a centre of rotational symmetry (C) results in a cone point at C. (b) The symmetrically equivalent edges $\mathrm{CA}$ and $\mathrm{CB}$ that bound an asymmetric domain of the rosette pattern are glued to form the cone. (c) A modified pattern, that contains 5 mirror lines, intersecting at an order- 5 cone point. (d) Cutting the pattern along all mirrors results in the disc-like asymmetric domain illustrated, bounded by a pair of mirror lines that meet at $\mathrm{C}$, forming a $\star 5$ corner point.

$\mathbb{E}^{2}$ can be embedded in $\mathbb{E}^{3}$ without any deformation of their (uniform positive and zero) Gaussian curvature, $\mathbb{H}^{2}$ cannot. Generic hyperbolic surfaces in $\mathbb{E}^{2}$ therefore necessarily have singularities and/or curvature variations. TPMSs afford the simplest examples of such embeddings. Admissible hyperbolic orbifolds that embed to form (three-dimensional) crystalline patterns, with three $\left(\mathbb{E}^{3}\right)$ lattice vectors corresponding to those of the underlying TPMS, are a subset of all hyperbolic orbifolds. We call those cases crystallographic hyperbolic orbifolds. ${ }^{2}$ Thus, for example, $\star 23 \mathrm{k}$ patterns are not crystallographic, for all $k>6$. In contrast, the related hyperbolic orbifold, $\star 246$, is realized on the simplest TPMSs, the $P, D$ and Gyroid surfaces (Robins et al., 2004a); $\star 246$ is therefore a crystallographic hyperbolic orbifold. Symmetry mutation of the $\mathrm{C}_{60}$ and graphene nets from $* 235$ and $\star 236$, respectively, to a 246 pattern, giving a crystalline (6.8.8) net, is possible. Indeed, three distinct nets are formed, one on each of these TPMSs, catalogued in the EPINET database as sqc9265, sqc12886 and sqc9271, respectively (Hyde et al., 2010) and labelled in the RCSR database as pcu-f, pbg and pbz (O'Keeffe et al., 2008). These 'polybenzene' nets describe the

\footnotetext{
${ }^{2}$ Mathematicians call any discrete group of isometries of the hyperbolic plane a 'non-euclidean crystallographic group' (Ratcliffe, 1994). We use a more restrictive sense of 'crystallographic' in this article to denote two-dimensional hyperbolic symmetries that are compatible with three-dimensional euclidean space groups.
}

bonding networks of hypothetical hyperbolic graphenes, or schwarzites (Lenosky et al., 1992). Their relation to conventional graphene and fullerene structures can be described in structural terms by disclinations (Lijima et al., 1992). Disclinations, which modify the rotational symmetry, also edit the curvature of a pattern: an operation that is equivalent to the symmetry mutation procedure.

Without a unified naming schema for groups of isometries of all three two-dimensional spaces, the relation between elliptic, flat and hyperbolic patterns is far from evident. While we do not expect crystallographers to give up their familiar nomenclature for plane and point groups readily, there is a good case for doing just that, given the power and clarity of Conway's naming schema for two-dimensional orbifolds.

The article is structured as follows. We first outline the naming schema briefly, and suggest a generic taxonomy of all two-dimensional groups into seven distinct classes, that allows us to tame somewhat the huge proliferation of hyperbolic groups. Those classes emerge naturally from the orbifold approach, since they correspond (principally) to distinct orbifold topologies. Lastly, we tabulate the crystallographic hyperbolic orbifolds related to all known genus-three TPMSs (the $P$, Gyroid, D, CLP and $H$ surfaces) and the single genusfour TPMS (the $I-W P$ surface), and list the resulting space groups formed by embedding those orbifolds on these surfaces.

\section{Orbifold features: cones, corners, handles and cross- caps}

Consider a symmetric decoration of $\mathbb{S}^{2}, \mathbb{E}^{2}$ or $\mathbb{H}^{2}$ that is formed by a discrete group of isometries (Stillwell, 1992). A fundamental domain (or asymmetric unit) can be traced in the surface such that all points within the domain are symmetrically distinct. A set of two-dimensional isometries are required to generate the complete pattern, possibly including combinations of centres of rotational symmetry, mirror lines of reflection symmetry, and glide lines combining reflections with translations, and pure translations. The symmetry operations can also be interpreted as rules for gluing the fundamental domain into an orbifold. The topology of a compact orbifold can be summarized succinctly by listing four types of topological module which must be glued onto or excised from a sphere to create that orbifold. The orbifold then is a quotient space of the general plane (elliptic, flat or hyperbolic) factored by the isometries of the pattern. A fuller description can be found elsewhere; here we give only a brief outline to allow explication of our proposed orbifold taxonomy (Stillwell, 1992; Conway et al., 2008).

A simple example is the asymmetric domain of a rotationally symmetric rosette pattern (of order A say), Fig. 2. It is a radial sector based at the centre of rotational symmetry, with apex angle $2 \pi / A$. The sector is bounded by two lines; points on either line equidistant from the centre are related to each other by the rotation isometry and they are glued together. The wedge-shaped asymmetric domain becomes a cone, whose apex or 'cone point' is the centre of symmetry, illustrated in 
Fig. 2. A centre of rotational symmetry in a group with compact fundamental domain generates a singular point in the orbifold and appears as an integer A in the orbifold symbol.

Mirror lines result in a topological feature of orbifolds, namely boundaries. If $a$ mirror lines intersect at a point, they generate a singular corner point on the boundary with (internal surface) angle $\pi / a$. For example, a rosette orbifold formed by intersecting mirrors in Fig. 2(c) is a radial sector with angle $\pi / 5$ and is labelled by the symbol $\star 5$. In a compact orbifold, mirror lines must be loops that may or may not have corner points. Each boundary loop is designated explicitly in the orbifold symbol with a $\star$ ('star'), followed by the orders of the corner points, listed in cyclic order. The kaleidoscopic group of the graphene net, bounded by mirror lines intersecting at three corners with angles $\pi / 2, \pi / 3$ and $\pi / 6$ is $\star 236$. A corner-free loop is labelled $\star$ (equivalent to $\star 1 \cdots 1$ since 1 entries are redundant).

The rosette example of Fig. 2 is worth exploring a little more carefully. Does a compact orbifold with a single cone or corner point, such as 5 or $\star 5$, denote a valid symmetry group of $\mathbb{S}^{2}, \mathbb{E}^{2}$ or $\mathbb{H}^{2}$ ? Clearly it cannot be a symmetry of $\mathbb{S}^{2}$, since a single cone or corner will necessarily have a twin cone or corner, located at its antipode, forming 55 or $\star 55$ orbifolds. A single rotational or roto-reflection symmetry in $\mathbb{E}^{2}$ or $\mathbb{H}^{2}$ has a fundamental domain that extends to infinity, so the orbifold cannot be compact. This observation implies a simple constraint on possible orbifold symbols: if they consist of just a single pair of corners or a pair of cones, they must be equivalent, viz. $\mathrm{AB}$ and $\star \mathrm{ij}$ imply $\mathrm{A}=\mathrm{B}$ and $\mathrm{i}=\mathrm{j}$, and the twodimensional pattern is an elliptic one (Conway et al., 2008).

These examples demonstrate a central Bauplan of all compact orbifolds. They can be constructed by starting with a sphere, $\mathbb{S}^{2}$, and, if relevant, pushing out cone points that encode centres of rotational symmetry (not on mirrors). Reflections are constructed by cutting the orbifold along (possibly polygonal) boundary loops (whose vertices define intersecting mirror edges), and deleting the (polygonal) patch, leaving a bounded orbifold. Additional symmetry elements of the group are formed by first excising caps from the sphere; the resulting manifold is a sphere with a number of (typically polygonal) boundaries, one for each removed cap. Additional topological modules can then be grafted to the punctured sphere along those loops. Those modules are of two types: handles (orbifold symbol $\circ$ ) or cross-caps $(\times)$, introduced below. Remaining boundaries encode mirror lines, with corners of angle $\pi / i$ at roto-reflection sites common to $i$ mirrors.

Recall that just four distinct symmetry operations are possible in two dimensions: rotations, reflections, glide reflections and translations. The first two operations are encoded in orbifolds by cone and corner points along boundaries, described above. The final two operations, translations and glide reflections, are signalled by non-trivial topology of the orbifold (i.e. a non-contractible loop) in an oriented or non-orientable orbifold, respectively.

A single translation operation induces a 1-periodic frieze pattern. The infinite periodic frieze pattern therefore maps to

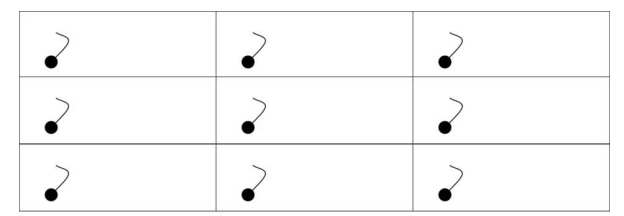

(a)

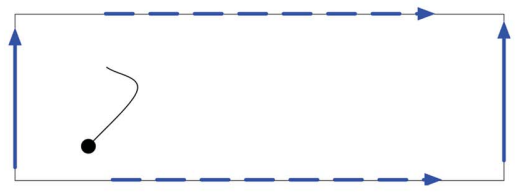

(b)

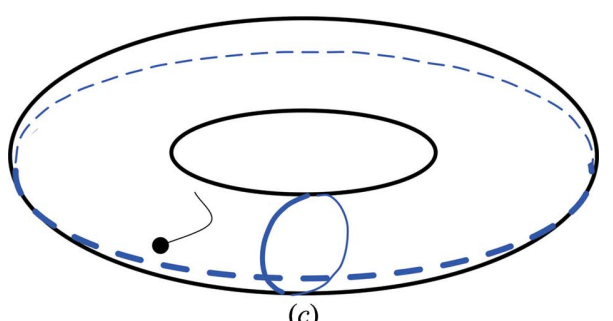

Figure 3

(c)

(a) A 2-periodic pattern in the euclidean plane, $\mathbb{E}^{2}$, with symmetry $p 1$. (b) A single asymmetric domain of the pattern, the unit cell, with the pair of translation vectors marked as blue lines. (c) Gluing opposite edges of the asymmetric domain related by lattice vectors to form the orbifold corresponding to $p 1$ results in a toroidal orbifold, denoted $\circ$.

a patterned cylinder under gluing of symmetrically equivalent sites in the frieze. A single equatorial loop around the cylinder corresponds to the lattice vector, glued head to tail. In the absence of any further isometries apart from a single translation, this simplest frieze pattern [with crystallographic friezegroup symbol $p 1$ (Kopsky \& Litvin, 2010)] maps to an infinitely extended cylinder devoid of any internal symmetries. The orbifold related to this pattern has symbol $\infty \infty$, corresponding to a pair of rotation centres of infinite order. We can understand this by analogy with the 55 orbifold derived from $\mathbb{S}^{2}$ due to a pair of cones of order 5 located at the north and south poles of $\mathbb{S}^{2}$ (derived from the fivefold rosette pattern of Fig. 2). This orbifold is formed by a fivefold pattern around the equator of $\mathbb{S}^{2}$; more generally AA generates $A$ copies of a motif around an equatorial belt of $\mathbb{S}^{2}$. A frieze (with crystallographic frieze-group symbol $p 1$ ) leads also to an AA orbifold, where the index A diverges, forming the $\infty \infty$ case.

Now consider the orbifold associated with a 2-periodic planar pattern, with symmetry $p 1$ (Fig. $3 a$ ). Since the pattern has translational symmetry only, its asymmetric domain is equivalent to the unit cell of the pattern (Fig. 3b). Gluing points related by one lattice vector forms a cylinder, decorated with a 1-periodic pattern due to the second lattice vector. The cylinder must therefore be glued end-to-end to itself, identifying points related by the second lattice vector, resulting in a bagel-shaped torus, whose two independent loops around the equatorial and meridional planes result from the pair of lattice vectors (Fig. 3c).

We call the module associated with the torus a 'handle', with orbifold symbol $\circ$ (Fig. 4a). Handles are attached to orbifolds by first excising discs from both the basic sphere 


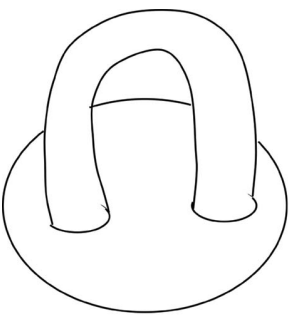

(a)

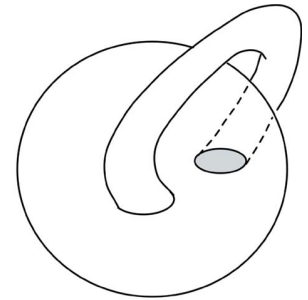

(b)
Figure 4

(a) A handle module, $\circ$, formed by removing a disc from the torus. (b) A cross-handle module, $\star x \times$, equivalent to a punctured Klein bottle.

around which the orbifold is constructed $(1 \rightarrow 1 \star=1 \star)$ and a torus $(\circ \rightarrow \circ \star)$, and then grafting these together by gluing the $(\star)$ boundaries generated by those excisions. We can write this operation thus: $\star \oplus \circ \star \rightarrow \circ$, where $\oplus$ denotes the gluing procedure.

Clearly, plane groups (acting in $\mathbb{E}^{2}$ ) are 2-periodic. However, groups of $\mathbb{H}^{2}$ can have an arbitrary number of independent translations $(>2)$. In general, a $2 n$-periodic pattern without additional isometries results in an orbifold with $\mathrm{n}$ handles, denoted $\circ \circ \cdots \circ\left(\right.$ or $\left.\circ^{\mathrm{n}}\right)$.

The most subtle isometry is that formed by a glide reflection. This too is encoded by a topological module in the orbifold. A non-contractible loop in a non-orientable manifold induces a glide reflection in the symmetry pattern. This can be demonstrated by a relatively simple example. The most familiar non-orientable manifold is the Möbius band, a onesided surface with just a single boundary edge. This orbifold emerges from gluing identical points within a fundamental domain of a crystalline pattern in $\mathbb{E}^{2}$ with symmetry group $\mathrm{cm}$, as shown in Fig. 5. The associated topological module that encodes glides is the 'cross-cap', with orbifold symbol $\times$ ('cross'). The Möbius band, which has a single mirror boundary, is therefore denoted $\star x$. Grafting this to an otherwise feature-free orbifold generates the boundary-free 'crosssurface': $\star \oplus \star x \rightarrow \times$. [Following Conway's convention, we

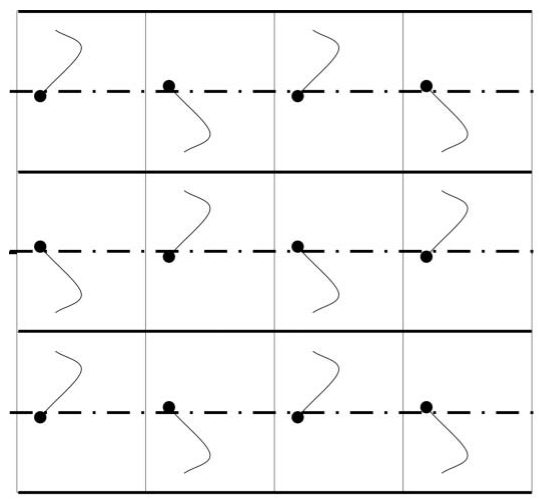

(a)

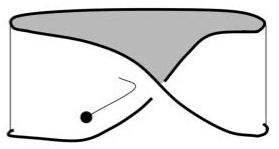

(b)
Figure 5

(a) A planar symmetric pattern with symmetry $\mathrm{cm}$. Horizontal mirrors and glide lines are indicated by full and dashed lines. (b) The Möbius strip $\star x$ is formed by gluing all symmetrically equivalent sites in the extended pattern to identical points. The single boundary loop in the Möbius strip corresponds to a mirror line. list all handle characters at the beginning of the orbifold word, and cross-caps at the end (Conway \& Huson, 2002).]

This non-orientable surface, also known as the projective plane, cannot be drawn in $\mathbb{E}^{3}$ without self-intersections. It is the simplest boundary-free non-orientable manifold. A well known result of topology is that a pair of Möbius bands grafted along their single boundary is the (non-orientable, boundary-free) Klein bottle, viz. $\star x \oplus \star x \rightarrow \times \times$. Like the cross-surface, the Klein bottle cannot be embedded in $\mathbb{E}^{3}$; selfintersections are inevitable. Removal of a disc results in a topological module that is the non-orientable analogue of the handle, namely the 'cross-handle' $(\star x \times)$, illustrated in Fig. 4(b).

Consider next the effect of attaching a handle $(\circ \star)$ to a Möbius band $(\star x)$. This can be 'crossed', forming a crosshandle, by sliding one end around the band, to form a cross-handle, $(\star \times \times \times)$, so that (Stillwell, 1980): $\diamond \star \oplus \star \times=$ $\circ \times=\times \times \times$.

The inter-conversion of handles and pairs of cross-handles (Fig. 4) implies that the presence of at least one non-orientable (cross-cap) module in an orbifold allows all orientable handles to be converted to pairs of cross-caps. So any non-orientable orbifold can be reduced to one with cross-caps only, via the following equality: $\circ^{\alpha} \cdots \times=\cdots \times^{2 \alpha+1}$.

\subsection{Building orbifolds from multiple features: a simple orbifold taxonomy}

In general, orbifolds contain an arbitrary number of features, made up of cones and corner points, and handles and cross-caps. Since the presence of any cross-caps in an orbifold allows handles to be morphed into pairs of cross-caps alone, we can simplify orbifold features further, so that nonorientable orbifolds contain cones, corners and cross-cap modules alone, and orientable orbifolds cones, corners and handles. In other words, orbifold features are either cones and corners, plus handles, or cones and corners and cross-caps.

With this simple toolkit of orbifold modules, any twodimensional discrete symmetric pattern can be characterized. Arbitrary combinations of these modules are allowed, with the exceptions listed above, namely the forbidden symbols $A, \star i$, $\mathrm{BC}$ and $\star \mathrm{jk}$ (unless $\mathrm{A}=\mathrm{i}=1$ or $\mathrm{B}=\mathrm{C}, \mathrm{j}=\mathrm{k}$ ) (Conway et al., 2008). Thus, the dictionary of two-dimensional orbifolds is vast, indeed multiply infinite, and just a fraction of words from that dictionary correspond to the groups known to crystallographers as point and plane groups. Given this richness, it is helpful to catalogue the dictionary.

First, we note that the orbifold dictionary contains three dialects related to the relevant spaces in which the orbifold words act: $\mathbb{S}^{2}, \mathbb{E}^{2}$ and $\mathbb{H}^{2}$. Following Conway (Conway \& Huson, 2002), we assign an Euler characteristic for each orbifold. Each feature - cones, corners, handles and cross-caps - has an associated characteristic. The characteristic associated with each feature is tabulated in Table 1.

These contributions to the orbifold characteristic can be deduced without difficulty from two-dimensional differential geometry and topology. The Euler characteristic of the orbi- 
Table 1

Contributions to the Euler characteristic $(\chi)$ of an orbifold due to all possible orbifold features (Conway, 1992).

All orbifolds contain a foundation sphere (whose symbol 1 is generally omitted); those corresponding to non-trivial symmetries also contain at least one of the additional features. Note that each $\star$ character in the orbifold word contributes -1 to the characteristic and associated corner points additional $(1-i) / 2 i$.

\begin{tabular}{llll}
\hline Isometry & Feature & $\begin{array}{l}\text { Orbifold } \\
\text { symbol }\end{array}$ & $\chi$ \\
\hline Trivial & Sphere & 1 & 2 \\
Pair of translations & Handle & $\circ$ & -2 \\
Rotation centre & Cone & $\mathrm{A}$ & $(1-\mathrm{A}) / \mathrm{A}$ \\
Reflection line & Boundary & $\star$ & -1 \\
Roto-reflection & Corner & $(\star) \mathrm{i}$ & $(1-\mathrm{i}) / 2 \mathrm{i}$ \\
Glide line & Cross-cap & $\times$ & -1 \\
\hline
\end{tabular}

fold is a simple sum of characteristics of individual features. The Euler characteristics of topological modules of orbifolds, namely the base sphere, plus additional mirrors, handles or cross-caps, follow from standard topology (Stillwell, 1980), with associated Euler characteristics of $-1,-2$ and -1 , respectively. We can determine characteristics of cone and corner points as follows. The Euler characteristic $(\chi)$ is related to the integral Gaussian curvature via (the global GaussBonnet theorem)

$$
2 \pi \chi=\iint K \mathrm{~d} a
$$

The integral curvature of cones and corners can be evaluated from the local Gauss-Bonnet theorem, that relates the curvature integrated over the surface area within a surface patch $\mathcal{P}$ bounded by a $p$-sided polygon with geodesic edges and internal vertex angles $v_{i}$ :

$$
\iint_{\mathcal{P}} K \mathrm{~d} a=(2-p) \pi+\sum_{i=1}^{p} v_{i}
$$

A cone point, $A$, is formed by wrapping identical points by the operation of an axis of A-fold rotational symmetry (Fig. 2). We can slice open the cone to give a pie-shaped asymmetric domain with apex angle $2 \pi / A$. It follows from equation (2) that the integral curvature associated with the cone point (concentrated at its apex) is $2 \pi / A$, compared with $2 \pi$ for a regular point (i.e. an order-1 cone point). The contribution of an order-A cone point to the Euler characteristic is therefore [from equation (1)] $(1 / A-1)$ or $-(A-1) / A$. The contribution of corners to the Euler characteristic also follows from equation (2). Consider a polygon of mirror lines, $\star \mathrm{ij} \cdots \mathrm{k}$. This can be formed by excising a polygonal face from the orbifold, i.e. a geodesic polygon with vertex angles $\pi / \mathrm{i}, \pi / \mathrm{j} \ldots \pi / \mathrm{k}$. The 'lost' integral curvature is therefore

$$
2 \pi-\left(\pi-\frac{\pi}{\mathrm{i}}+\pi-\frac{\pi}{\mathrm{j}}+\ldots+\pi-\frac{\pi}{\mathrm{k}}\right) .
$$

Equation (1) implies that the Euler characteristic of the orbifold is reduced by

$$
1-\left(\frac{i-1}{2 i}+\frac{j-1}{2 j} \ldots+\frac{k-1}{2 k}\right) .
$$

The total Euler characteristic for an orbifold containing an arbitrary number of cones, corners and handles or cross-caps is determined by adding all associated characteristics, including that of the foundational form for the orbifold, a sphere. The characteristic of an orientable orbifold, of the form ${ }^{\alpha} \mathrm{AB} \cdots \mathrm{C} \star \mathrm{ij} \cdots \mathrm{k} \cdots \star \operatorname{lm} \cdots$ is therefore

$$
\begin{aligned}
\chi= & 2-\left\{2 \alpha+\frac{A-1}{A}+\frac{B-1}{B} \cdots+\frac{C-1}{C}+\right. \\
& 1+\frac{i-1}{2 i}+\frac{j-1}{2 j}+\cdots \frac{k-1}{2 k} \cdots \\
& \left.+1+\frac{l-1}{2 l}+\frac{m-1}{2 m}+\cdots\right\} .
\end{aligned}
$$

Likewise, the characteristic of a non-orientable orbifold, of the form $A B \cdots C \star \mathrm{ij} \cdots \mathrm{k} \cdots \star \operatorname{Im} \cdots \times^{\beta}$, is

$$
\begin{aligned}
\chi= & 2-\left\{\frac{A-1}{A}+\frac{B-1}{B} \cdots+\frac{C-1}{C}+\right. \\
& 1+\frac{i-1}{2 i}+\frac{j-1}{2 j}+\cdots \frac{k-1}{2 k} \cdots \\
& \left.+1+\frac{I-1}{2 l}+\frac{m-1}{2 m}+\cdots+\beta\right\} .
\end{aligned}
$$

The orbifold characteristic is a very useful index that allows the orbifold dictionary to be readily split into its three constituent dialects, belonging to $\mathbb{S}^{2}, \mathbb{E}^{2}$ and $\mathbb{H}^{2}$. Recall that two-dimensional point groups act on $\mathbb{S}^{2}$, a space of constant positive Gaussian curvature, plane groups act on $\mathbb{E}^{2}$ (zero Gaussian curvature) and hyperbolic groups act on $\mathbb{H}^{2}$, a space of constant negative Gaussian curvature. Since the orbifold characteristic scales with the integral (Gaussian) curvature of the orbifold [equation (1)], the relevant space associated with an orbifold is given by the sign of the characteristic. In other words, if $\chi$ is positive, the isometries act on the sphere, if it is zero, they act on the euclidean plane. Thus, orbifolds of positive characteristic are point groups, those of zero characteristic are plane groups. Finally, if the orbifold characteristic is negative, the isometries act on the hyperbolic plane and the orbifold belongs to a two-dimensional hyperbolic group.

Notice that Table 1 assigns negative characteristics to all orbifold features, except the foundation sphere. The length of orbifold words is unbounded. Thus a generic orbifold symbol apart from those with very short words - will induce a negative characteristic. In other words, most two-dimensional orbifolds are hyperbolic. This is due to the vast number of possible isometries of the hyperbolic plane compared with those on the sphere or flat plane. In order to catalogue that wealth more systematically, we introduce seven distinct orbifold classes in the next section.

\section{Orbifold classes}

The previous exposition of orbifolds, their features and their associated two-dimensional geometries is essentially that 
developed by Conway and colleagues. Here we delve further into the topological structure of orbifolds, and introduce distinct classes to aid our analysis of point and plane groups as well as hyperbolic examples.

We first distinguish between simply connected and multiply connected orbifolds. To do that, ignore all integer cone and corner indices (equivalent to setting them to 1 ), and consider only their topological features, i.e. $\star, \circ$ or $\times$ characters. Simply connected examples include orbifolds with no topological features, and those whose words contain just one $\star$ character. All remaining orbifolds are multiply connected. That includes all orbifolds whose words contain $\circ$ and $\times$ characters, multiple $\star$ characters, and mixed cases, containing both $\circ$ and $\star$ or $\times$ and $\star$ characters.

Both simply and multiply connected orbifolds are classified further. First, we distinguish closed (boundary-free) orbifolds from those with (possibly multiple) boundaries.

Two distinct classes of simply connected orbifolds with boundary are flagged: coxeter and hat orbifolds. Coxeter orbifolds are topological discs (Fig. 6), with possible corners but without interior cones; they are named after H. S. M. Coxeter, who pioneered the study of discrete reflection groups (kaleidoscopes), now known as Coxeter groups (Coxeter, 1934). Coxeter orbifolds have words of the form $\star i j \cdots$. Hat orbifolds also contain a single boundary loop, and at least one

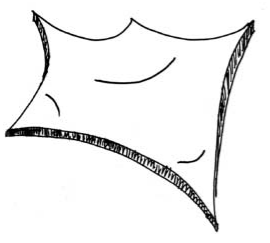

C

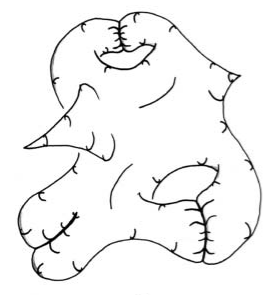

P

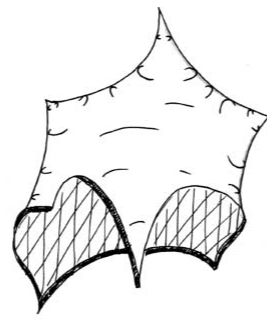

$\mathrm{H}$

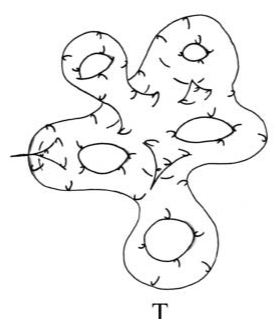

$\mathrm{T}$

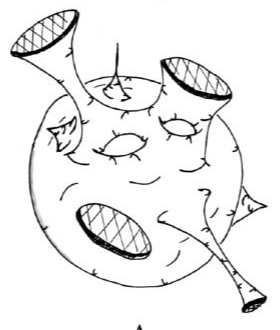

A
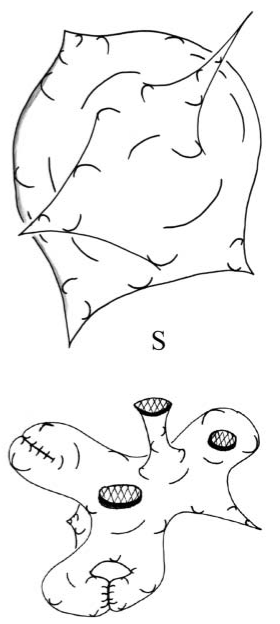

M
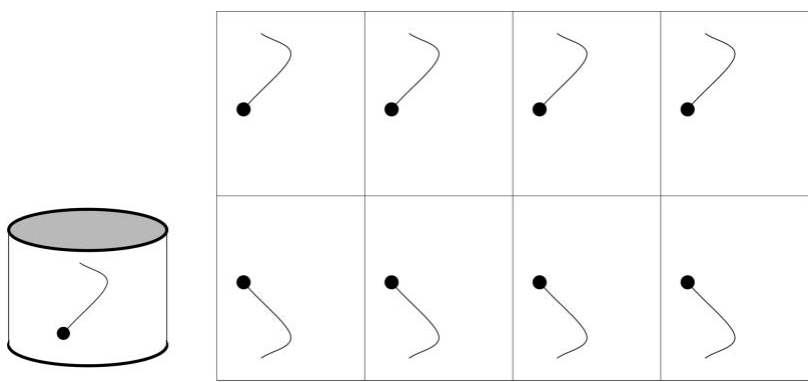

Figure 7

Printing (left) a decorated $\star \star$ (annular) orbifold, forming (right) a symmetric planar pattern with symmetry $\mathrm{pmm}$.

non-trivial cone point: their simplest members are orbifolds of the form $A \star$, which resemble dunce caps. [Generic members resemble yet more exotic hats (Fig. 6).] Since generic orientable, boundary-free, simply connected examples resemble inflated balloons with any number of cone singularities (Fig. 6 ), we call these examples stellate orbifolds. Three-pointed samosas (cooked in Indian kitchens) and four-pointed pillows demonstrate the forms of certain stellate orbifolds (with orbifold words $A B C$ and $A B C D$, respectively).

We classify multiply connected orbifolds into orientable and non-orientable classes, both with or without boundaries. We name these four multiply connected classes after the simplest representative manifolds. Thus, we call all multiply connected orientable orbifolds with boundaries annular, after the annulus ( $\star \star)$, the simplest example (Fig. 7). More general members in this class contain handles, additional boundaries and cones and corner points (Fig. 6). All annular examples can be represented as discs with bands, the standard form for orientable manifolds with boundaries (Stillwell, 1980). Nonorientable analogues of annular orbifolds are möbius orbifolds, named after the Möbius band, the simplest multiply connected non-orientable manifold with boundary. Generic möbius orbifolds may also contain additional boundaries, cross-caps as well as cone and corner points (Fig. 6) and can be canonically drawn as discs with twisted bands (Stillwell, 1980). Boundary-free multiply connected orbifolds are either toroidal or projective, depending on whether they are orientable or non-orientable. Orbifolds containing a single $x$ character only (and optional cones) resemble the projective plane (Fig. 6). Generic projective orbifolds have words of the form $A B \cdots \times \cdots \times$. The orbifold $\circ$ is the torus; generic toroidal examples contain multiple handles as well as cones.

This taxonomy is summarized in Table 2. The relevant class can be deduced from the orbifold word [possibly rewritten to remove mixed $\circ \times$ characters following the substitution rule of equation (2)] without difficulty according to the heuristic in Table 2. We have been using it for some years now, and have yet to find a more succinct and practical schema. As we shall show, it is particularly helpful when classifying the hyperbolic orbifolds, though it is a useful tool to explore relations between plane and point groups also. Indeed, this topological classification of symmetry classes affords a simple correspondence between symmetries of all two-dimensional reticula- 
Table 2

Orbifold classification schema into three simply connected and four multiply connected classes.

The relevant class for an arbitrary orbifold depends on the presence or absence of orbifold features in the

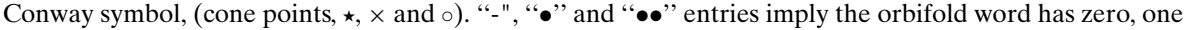
and two occurrences of the relevant feature, respectively. Bracketed entries (e.g. "(•)") denote optional additional features.

\begin{tabular}{llllllll}
\hline Connectivity & Class & Oriented & Bounded & $\circ$ & Cone pts. & $\star$ & $\times$ \\
\hline \multirow{2}{*}{ Simple } & coxeter & yes & yes & - & - & $\bullet$ & - \\
& stellate & yes & no & - & $\bullet \bullet(\bullet)$ & - & - \\
& hat & yes & yes & - & $\bullet(\bullet)$ & $\bullet$ & - \\
Multiple & projective & no & no & - & $(\bullet)$ & - & $\bullet(\bullet)$ \\
& möbius & no & yes & - & $(\bullet)$ & $\bullet(\bullet)$ & $\bullet(\bullet)$ \\
& annular† & yes & yes & $(\bullet)$ & $(\bullet)$ & $\bullet \bullet(\bullet)$ & - \\
& toroidal & yes & no & $\bullet(\bullet)$ & $(\bullet)$ & - & - \\
\hline
\end{tabular}

$\dagger$ All annular orbifolds have multiple mirrors, except $\circ \star$ or $\circ \mathrm{AB} \ldots \star \mathrm{ij} .$. , with only a single mirror.

extended pattern decorating $\mathbb{S}^{2}, \mathbb{E}^{2}$ or $\mathbb{H}^{2}$. This construction can be reversed, to form the complete symmetric pattern - in $\mathbb{S}^{2}, \mathbb{E}^{2}$ or $\mathbb{H}^{2}$ - from an orbifold decorated with a single copy of the asymmetric domain. Thus, orbifolds describe discrete symmetry groups of the three homogeneous twodimensional geometries. A useful way to imagine this process is to conceptualize the orbifold as a stamp, inked with the pattern contained within a single asymmetric domain. Bounded orientable orbifolds have two sides; we allow the ink to seep through to coat both sides of the orbifold. The complete extended

tions and orbifold topology. We propose those seven orbifold classes - coxeter, hat, stellate, projective, toroidal, möbius and annular - as a convenient taxonomic scheme for all discrete two-dimensional groups acting on the sphere (including crystallographic point groups), the euclidean plane (plane groups) and the hyperbolic plane. The following sentence is a useful aide memoire: 'Conway's symbols have pretty much all topologies.'

\subsection{Orbifold equivalence and canonical forms}

A single orbifold can often be described by a multiplicity of distinct orbifold words, related to each other by reordering the symbols and digits. Since the linear word describes features of a topological object, the ordering of those features is irrelevant. Following Conway, we list features in orientable orbifolds in the following order: (possibly multiple) handles (o), (possibly multiple) cones (A) appended by (possibly multiple) corners $(\star \mathrm{ij} \cdots)$. Non-orientable orbifolds are described by words whose features are ordered thus: cones, corners and cross-caps. The ordering of multiple cone points and multiple disjoint boundary loops is arbitrary (so that e.g. ABCD and ACBD are identical, as are $\star i \star j k$ and $\star j k \star i)$.

In general, the equivalence of different words is evident from the orbifold topology. For example, the words $\star 246, \star 462$, $\star 624, \star 264, \star 426$ and $\star 642$ encode the same orbifold. Since each sequence of corner points encodes the same triangle, they are equivalent. (Recall that corner points are listed in cyclic order around the $\star$ boundary.) In contrast, $\star 2346$ and $\star 3246$ are not equivalent, since they describe different quadrilaterals. In general a mirror boundary loop $\star i j k \ldots l$ is equivalent to all cyclic (forward and reverse) permutations of the corners. (We prefer a canonical form for these boundary loops that chooses the permutation that starts with the smallest corner index, followed by indices that are also as low as possible consistent with a cyclic trajectory around the loop.)

\section{Printing orbifolds}

Our construction of orbifolds described above emerges from gluing of symmetrically identical sites in the complete symmetric pattern is printed from the single motif decorating the orbifold by rolling the orbifold on the relevant twodimensional substrate $\left(\mathbb{S}^{2}, \mathbb{E}^{2}\right.$ or $\left.\mathbb{H}^{2}\right)$, thereby printing the pattern. This process of printing from the orbifold to the 'universal cover' is analogous to printing a frieze pattern from a cylindrical seal by endless rolling about the axis of the cylinder. Thus, for example, printing around a cone point of order $A$ on an orbifold generates a centre of $A$-fold rotational symmetry (whose order depends on the apex angle of the cone) by rolling about the cone tip (cf. Fig. 2).

Printing the orbifold over a boundary (mirror) edge is done by first printing one side, then flipping the orbifold through the edge to print the other side. The orbifold prints a reflected copy once the stamp is turned over through the boundary [a nice animation of this process can be viewed within the Simetria DVD, available from Atractor (2010) (see also Conway et al., 2008)]. An example of this process is the extension from the $\star \star$ (annular) orbifold to $\mathbb{E}^{2}$, generating the two-periodic plane group, illustrated in Fig. 7. The annular orbifold can be rolled along one direction to give a frieze pattern. An adjacent frieze (mirror reversed) results by turning the orbifold inside out across an edge and rolling that reversed pattern, printing the inside. Repeated application of those operations gives the complete $\star \star$ or pmm design in $\mathbb{E}^{2}$. It is instructive to compare the pattern printed by $\star \star$ with its counterpart from $\star \times, c f$. Fig. 5 .

The relevant 'plane' that the orbifold prints out $\left(\mathbb{S}^{2}, \mathbb{E}^{2}\right.$ or $\mathbb{H}^{2}$ ) is determined by the sign of the orbifold characteristic, discussed in $\$ 2.1$. Since the orbifold carries an inherent metric determined by this sign, the orbifold is in intimate contact with the relevant uniformly curved two-dimensional space, printing a local patch as it rolls across its universal cover.

\subsection{Point and plane groups}

Since conventional three-dimensional euclidean point groups necessarily contain a fixed point, they correspond to discrete groups of isometries of $\mathbb{S}^{2}$. Further, two-dimensional plane groups are discrete groups of $\mathbb{E}^{2}$. There is a one-to-one correspondence between two-dimensional orbifolds and twodimensional discrete groups of $\mathbb{S}^{2}, \mathbb{E}^{2}$ or $\mathbb{H}^{2}$. Since we can 
Table 3

Two-dimensional plane groups: isometries of the euclidean plane, $\mathbb{E}^{2}$.

\begin{tabular}{lll}
\hline Class & $\begin{array}{l}\text { Orbifold } \\
\text { symbol }\end{array}$ & $\begin{array}{l}\text { Crystallographic } \\
\text { symbol }\end{array}$ \\
\hline coxeter & $\star 632$ & $p 6 m$ \\
& $\star 442$ & $p 4 m$ \\
& $\star 333$ & $p 3 m 1$ \\
stellate & $\star 2^{4}(\star 2222)$ & $p m m$ \\
& 632 & $p 6$ \\
& 442 & $p 4$ \\
hat & 333 & $p 3$ \\
& $2^{4}(2222)$ & $p 2$ \\
& $4 \star 2$ & $p 4 g$ \\
projective & $3 \star 3$ & $p 31 m$ \\
& $2 \star 22$ & $\mathrm{cmm}$ \\
toroidal & $22 \star$ & $\mathrm{pmg}$ \\
möbius & $22 \times$ & $\mathrm{pgg}$ \\
annular & $\times \times$ & $\mathrm{pg}$ \\
& $\circ$ & $\mathrm{pm}$ \\
& $\star \times$ & $\mathrm{cm}$ \\
& $\star \star$ & $\mathrm{pm}$ \\
\hline
\end{tabular}

determine at once whether an orbifold's universal cover is $\mathbb{S}^{2}$, $\mathbb{E}^{2}$ or $\mathbb{H}^{2}$, enumeration of point and plane groups is a simple matter of enumerating all orbifold words with non-negative characteristic (Conway \& Huson, 2002). It is an interesting exercise to explore this route to enumeration of point and plane groups, recommended to the reader. For example, it follows from the formulae for the orbifold characteristic that coxeter orbifolds of the form $\star v_{1} v_{2} \cdots v_{p}$ define spherical, planar and hyperbolic kaleidoscopes where

$$
(2-p) \prod_{i=1}^{p}\left(\mathrm{v}_{i}\right)+\sum_{i=1}^{p}\left(\frac{\prod_{j=1}^{p}\left(\mathrm{v}_{j}\right)}{\mathrm{v}_{i}}\right)
$$

is positive, zero or negative, respectively. Thus, any orbifold of the form $\star v_{1} v_{1}(p=2)$ is spherical and defines a point group. Conversely, once $p>4$, all coxeter orbifolds are necessarily hyperbolic. Planar coxeter orbifolds obey the relation

$$
\sum_{i=1}^{p}\left(\frac{1}{v_{i}}\right)=p-2,
$$

which has (positive integer) solutions only for $p=3$, 4. All remaining coxeter orbifolds, with generic words $\star v_{1} v_{2} \cdots v_{p}$, are hyperbolic. Similar relations can be deduced readily for all simply connected orbifolds, using the formulae in Table 1.

We use those relations to enumerate complete catalogues of point and plane groups that have positive and zero characteristics, respectively. We have listed orbifolds of $\mathbb{E}^{2}$ and $\mathbb{S}^{2}$ and their corresponding point and plane groups in Tables 3 and 4 , respectively.

\subsection{Group-subgroup orders and ranking non-euclidean groups}

The magnitude of an orbifold characteristic, $|\chi|$, is informative for spherical and hyperbolic cases. Since the orbifold acts on spaces of constant (positive or negative) curvature, the area of an orbifold scales with the magnitude of its characteristic via equation (1). This area is equal to that of a single asymmetric domain of the group in its universal cover $\left(\mathbb{H}^{2}\right.$ or
Table 4

Two-dimensional spherical orbifolds and corresponding two-dimensional point groups of their universal covers on the sphere, $\mathbb{S}^{2}$.

Group orders and orbifold characteristics $(\chi)$ are also tabulated. The ordering of integer (rotational symmetry) entries in orbifold symbols for coxeter and stellate orbifolds on $\mathbb{S}^{2}$ are flexible; we adopt the convention of listing integers in ascending order. Conventional crystallographic (abbreviated HermannMauguin) and Schönflies symbols are listed for the point groups. $k$ denotes any

\begin{tabular}{|c|c|c|c|c|c|}
\hline Class & Orbifold & $\begin{array}{l}\text { Hermann- } \\
\text { Mauguin }\end{array}$ & Schönflies & Order & $\chi$ \\
\hline \multirow[t]{14}{*}{ coxeter } & $\star 235$ & - & $I_{h}$ & 120 & $\frac{1}{60}$ \\
\hline & $\star 234$ & $m \overline{3} m$ & $O_{h}$ & 48 & $\frac{1}{24}$ \\
\hline & $\star 233$ & $\overline{4} 3 m$ & $T_{d}$ & 24 & $\frac{1}{12}$ \\
\hline & $\star 22 \mathrm{k}$ & - & $D_{k h}$ & $4 k$ & $\frac{1}{2 k}$ \\
\hline & $\star 226$ & $6 / \mathrm{mmm}$ & $D_{6 h}$ & 24 & $\begin{array}{l}\frac{1}{12} \\
12\end{array}$ \\
\hline & $\star 224$ & $4 / \mathrm{mmm}$ & $D_{4 h}$ & 16 & $\frac{1}{8}$ \\
\hline & $\star 223$ & $\overline{6} 2 m$ & $D_{3 h}$ & 12 & $\frac{1}{6}$ \\
\hline & $\star 222$ & $\mathrm{mmm}$ & $D_{2 h}$ & 8 & $\frac{1}{4}$ \\
\hline & $\star \mathrm{kk}$ & - & $C_{k v}$ & $2 k$ & $\begin{array}{l}\frac{4}{k} \\
\frac{1}{k}\end{array}$ \\
\hline & $\star 66$ & $6 \mathrm{~mm}$ & $C_{6 v}$ & 12 & $\frac{1}{6}$ \\
\hline & $\star 44$ & $4 m m$ & $C_{4 v}$ & 8 & $\begin{array}{l}6 \\
\frac{1}{4}\end{array}$ \\
\hline & $\star 33$ & $3 m$ & $C_{3 v}$ & 6 & $\frac{1}{3}$ \\
\hline & $\star 22$ & $m m 2$ & $C_{2 v}$ & 4 & $\frac{1}{2}$ \\
\hline & $\star$ & $m$ & $C_{s}$ & 2 & 1 \\
\hline \multirow[t]{13}{*}{ stellate } & 235 & - & $I$ & 60 & $\frac{1}{30}$ \\
\hline & 234 & 432 & $O$ & 24 & $\frac{1}{12}$ \\
\hline & 233 & 23 & $T$ & 12 & $\begin{array}{l}\frac{1}{6} \\
\end{array}$ \\
\hline & $22 \mathrm{k}$ & - & $D_{k}$ & $2 k$ & $\frac{1}{k}$ \\
\hline & 226 & 622 & $D_{6}$ & 12 & $\begin{array}{l}\frac{\pi}{6} \\
\frac{1}{6}\end{array}$ \\
\hline & 224 & 422 & $D_{4}$ & 8 & $\frac{1}{4}$ \\
\hline & 223 & 32 & $D_{3}$ & 6 & $\frac{1}{3}$ \\
\hline & 222 & 222 & $D_{2}$ & 4 & $\frac{1}{2}$ \\
\hline & $\mathrm{kk}$ & - & $C_{k}$ & $k$ & $\frac{2}{k}$ \\
\hline & 66 & 6 & $C_{6}$ & 6 & $\begin{array}{l}\frac{\pi}{3} \\
\end{array}$ \\
\hline & 44 & 4 & $C_{4}$ & 4 & $\frac{3}{2}$ \\
\hline & 33 & 3 & $C_{3}$ & 3 & $\frac{2}{3}$ \\
\hline & 22 & 2 & $C_{2}$ & 2 & 1 \\
\hline \multirow[t]{9}{*}{ hat } & $3 \star 2$ & $m \overline{3}$ & $T_{h}$ & 24 & $\frac{1}{12}$ \\
\hline & $2 \star k$ & - & $D_{k d}$ & $4 k$ & $\frac{1}{2 k}$ \\
\hline & $2 \star 3$ & $\overline{3} m$ & $D_{3 d}$ & 12 & $\frac{1}{6}$ \\
\hline & $2 \star 2$ & $\overline{4} 2 m$ & $D_{2 d}$ & 8 & $\frac{1}{4}$ \\
\hline & $k_{\star}$ & - & $C_{k h}$ & $2 k$ & $\frac{1}{k}$ \\
\hline & $6 \star$ & $6 / m$ & $C_{6 h}$ & 12 & $\begin{array}{l}\frac{\pi}{6} \\
\frac{1}{6}\end{array}$ \\
\hline & $4 \star$ & $4 / m$ & $C_{4 h}$ & 8 & $\frac{1}{4}$ \\
\hline & $3 \star$ & $\overline{6}$ & $C_{3 h}$ & 6 & $\frac{4}{3}$ \\
\hline & $2 \star$ & $2 / m$ & $C_{2 h}$ & 4 & $\frac{1}{2}$ \\
\hline \multirow[t]{5}{*}{ projective } & $(2 k+1) \times$ & - & $C_{k i}$ & $4 k+2$ & $\frac{1}{2 k+1}$ \\
\hline & $3 x$ & $\overline{3}$ & $C_{3 i}$ & 6 & $\frac{1}{3}$ \\
\hline & $\times$ & $\overline{1}$ & $C_{i}$ & 2 & 1 \\
\hline & $(2 k) \times$ & - & $S_{k}$ & $4 k$ & $\frac{1}{2 k}$ \\
\hline & $2 \times$ & $\overline{4}$ & $S_{4}$ & 4 & $\begin{array}{l}2 k \\
\frac{1}{2}\end{array}$ \\
\hline
\end{tabular}
positive integer.

Table 5

Frieze groups: one-periodic isometries of $\mathbb{E}^{2}$.

\begin{tabular}{lll}
\hline Class & $\begin{array}{l}\text { Orbifold } \\
\text { symbol }\end{array}$ & $\begin{array}{l}\text { Crystallographic } \\
\text { symbol }\end{array}$ \\
\hline coxeter & $\star 22 \infty$ & $p 2 m m$ \\
& $\star \infty \infty$ & $p 1 m 1$ \\
stellate & $22 \infty$ & $p 2$ \\
& $\infty \infty$ & $p 1$ \\
hat & $2 \star \infty$ & $p 2 m g$ \\
& $\infty \star$ & $p 11 m$ \\
projective & $\infty \times$ & $p 11 g$ \\
\hline
\end{tabular}


$\mathbb{S}^{2}$ ). The order of a group is thus coupled to the characteristic of the relevant orbifold. For example, subgroups with orbifold $\mathcal{S}$ of index $\alpha$ of a group with orbifold $\mathcal{G}$ have characteristic $\chi(\mathcal{S})=\alpha \chi(\mathcal{G})$. For example, the order of a two-dimensional point group with orbifold $\mathcal{P}$ is given by the number of copies of the asymmetric domain that cover the sphere once. Since $\mathbb{S}^{2}$ has integral curvature $4 \pi$, its characteristic is 2 and so the order of $\mathcal{P}$,

$$
\mathcal{O}(\mathcal{P})=\frac{2}{\chi(\mathcal{P})}
$$

We can generalize this to deduce the order of a hyperbolic group, whose orbifold $\mathcal{H}$ describes the isometries of a hyperbolic manifold $M$ of Euler-Poincaré characteristic $\chi(M)$ :

$$
\mathcal{O}(\mathcal{H})=\frac{\chi(M)}{\chi(\mathcal{H})}
$$

The orbifold characteristic therefore offers a convenient ranking of point and hyperbolic groups. For point groups, this index is equal to the number of isometries in the group (Conway et al., 2008). Point groups can be found for arbitrarily small $\chi$, due to the orbifolds with arbitrarily high-order cone or corner points $($ e.g. $\mathrm{NN}, \star 22 \mathrm{~N}$, where $\mathrm{N}$ is any positive integer). Lunes or triangles of arbitrarily small area are therefore valid asymmetric domains on $\mathbb{S}^{2}$.

All plane groups have zero characteristic. Since they lie in $\mathbb{E}^{2}$, which has vanishing Gaussian curvature, it follows from equation (1) that their area is independent of characteristic. Indeed, in contrast to elliptic and hyperbolic patterns, planar euclidean patterns can be homothetically rescaled while preserving all isometries, so the area of asymmetric domains of symmetric patterns of $\mathbb{E}^{2}$ is free.

In contrast to symmetric patterns on $\mathbb{S}^{2}$ and $\mathbb{E}^{2}$, there is a strict lower bound on the area of an asymmetric domain that gives a symmetric pattern on $\mathbb{H}^{2}(1 / 84)$. Since the area of a single asymmetric domain of the group in $\mathbb{H}^{2}$ scales linearly with the magnitude of the orbifold characteristic, we can rank hyperbolic groups in descending order of their (negative) characteristics, from the highest symmetry (least negative characteristic) to the lowest, analogous to a ranking of point groups according to their group order. The maximal characteristic for a hyperbolic orbifold is $-1 / 84$, realized by the Coxeter orbifold $\star 237$. This group is therefore the most symmetric pattern in $\mathbb{H}^{2}$. A list of other very symmetric examples is given in Table 6, including the most symmetric cases of each of the seven orbifold classes. The observant reader will notice that the ordering of our orbifold classes, viz. coxeter, stellate, hat, projective, möbius, annular, toroidal, corresponds to the ranking from most to least symmetric of the most symmetric example of each class.

\section{Periodic groups}

\subsection{0-, 1- and 2-periodic groups}

We can further classify all orbifolds by the number of independent translation vectors that are formed by printing the orbifold.
Crystallographers often distinguish between crystallographic point groups, that allow only rotations of order 2,3 , 4 and 6 , and others. That restriction excludes orbifolds of $\mathbb{S}^{2}$ whose integer entries are not one of those allowed orders, leaving 32 instances among the infinite number of orbifolds listed in Table 4. These 32 examples define 0-periodic (crystallographic) groups in $\mathbb{E}^{3}$.

1-Periodic frieze groups in $\mathbb{E}^{3}$ emerge as limit cases of point groups or orbifolds on $\mathbb{S}^{2}$, with unbounded order. As discussed above, this follows since periodic friezes tile cylinders, or spheres of unbounded radius compared with the dimension of a frieze unit cell. Eight cases of point groups are listed in Table 4 with arbitrary integer indices, $k: \star 22 \mathrm{k}, \star \mathrm{kk}, 2 \star \mathrm{k}, \mathrm{k} \star, 22 \mathrm{k}, \mathrm{kk}$, $(2 k+1) \times$ and $(2 k) \times$. All of those form friezes in the limit $\mathrm{k} \rightarrow \infty$, giving the seven frieze groups listed in Table 5 (Conway et al., 2008).

2-Periodic groups in $\left(\mathbb{E}^{2}\right.$ or) $\mathbb{E}^{3}$ include all of the plane groups listed in Table 3 .

\subsection{3-Periodic groups: crystallographic two-dimensional hyperbolic groups}

3-Periodic patterns can be generated in $\mathbb{E}^{3}$ via embeddings of two-dimensional manifolds, provided those manifold embeddings are themselves 3-periodic. Consider, for illustrative purposes, possible embeddings of hyperbolic patterns whose orbifold is $\star 237$. This orbifold and associated groups have been explored since the pioneering group theoretic work of Klein in the 19th century; its symmetries are realized by the Klein quartic (Levy, 2001; Mackay, 1986; Baez, accessed 2013). No embedding in $\mathbb{E}^{3}$ maintains all the two-dimensional isometries of the $\star 237$ group. Similarly, the crystallographic restriction of rotation orders to 2,3,4 and 6 ensures that many other hyperbolic orbifolds cannot be embedded in $\mathbb{E}^{3}$ to form 3-periodic patterns. It is therefore of interest to determine which examples of hyperbolic orbifolds can be embedded in $\mathbb{E}^{3}$.

We define crystallographic hyperbolic groups ${ }^{3}$ to be those that can be realized by (hyperbolic) surfaces that are translationally symmetric in $\mathbb{E}^{3}$. Among the orbifolds listed in Table 6 , the most symmetric example that is known to be crystallographic is $\star 246$.

It is surely no coincidence that this hyperbolic twodimensional group is realized by the embeddings of the $P, D$ and Gyroid TPMSs, found in a variety of hard and soft condensed materials. ${ }^{4}$ The hyperbolic translational symmetries of these surfaces have orbifold $\circ \circ \circ$. It is therefore possible to embed surfaces in $\mathbb{E}^{3}$ whose orbifolds are subgroups of $\star 246$ and supergroups of $\circ \circ \circ$, defined by appropriate group relations described in detail elsewhere

\footnotetext{
${ }^{3}$ Note that we use this term in a different sense to that of Ratcliffe (1994).

${ }^{4}$ The intrinsic symmetries of the $\star 246$ group lift to euclidean symmetries for the $P$ and $D$ surfaces only. In the case of the Gyroid, the hyperbolic mirrors are not realized as euclidean symmetries in $\mathbb{E}^{3}$, whereas all the rotational symmetries are manifested as euclidean three-dimensional symmetries. Thus, though the intrinsic hyperbolic symmetry of the Gyroid is $\star 246$, only the symmetries of the (stellate) order-2 subgroup, 246, are evident in $\mathbb{E}^{3}$ (Robins et al., 2005; Ramsden et al., 2009), as discussed further in Appendix A1.
} 
Table 6

Ranking of two-dimensional hyperbolic orbifolds by decreasing characteristic $(\chi \geq-1 / 2)$.

The most symmetric examples of orbifolds from each of the seven classes classified according to Table 2 are in bold.

\begin{tabular}{|c|c|c|}
\hline$\chi$ & Orbifold & Class \\
\hline$-\frac{1}{84}$ & $\star 237$ & coxeter \\
\hline $\begin{array}{r}84 \\
-\frac{1}{48}\end{array}$ & $\star 238$ & coxeter \\
\hline $\begin{array}{r}48 \\
-\frac{1}{42}\end{array}$ & 237 & stellate \\
\hline$-\frac{1}{40}$ & $\star 245$ & coxeter \\
\hline $\begin{array}{r}40 \\
-\frac{1}{36}\end{array}$ & $\star 239$ & coxeter \\
\hline$-\frac{1}{30}$ & $\star 23(10)$ & coxeter \\
\hline$-\frac{50}{132}$ & $\star 23(11)$ & coxeter \\
\hline$-\frac{1}{24}$ & $\star 23(12), \star 246, \star 334$ & coxeter \\
\hline & 238 & stellate \\
\hline & $3 \star 4$ & hat \\
\hline $0^{\circ}$ & $\vdots$ & $\vdots$ \\
\hline \multirow[t]{4}{*}{$-\frac{1}{12}$} & $\begin{array}{l}\star 23 \infty, \star 24(12), \star 266, \star 336, \star 344 \\
\star 2223\end{array}$ & $\begin{array}{l}\text { coxeter } \\
"\end{array}$ \\
\hline & 23(12), 246, 334 & stellate \\
\hline & $3 \star 6,4 \star 3,6 \star 2$ & hat \\
\hline & $2 \star 23$ & $"$ \\
\hline & $\vdots$ & $\vdots$ \\
\hline \multirow[t]{4}{*}{$-\frac{1}{8}$} & $\begin{array}{l}\star 25(20), \star 26(12), \star 288, \star 33(12), \star 346, \star 444 \\
\star 2224\end{array}$ & $\begin{array}{l}\text { coxeter } \\
"\end{array}$ \\
\hline & $23(24), 248$ & stellate \\
\hline & $4 \star 4,8 \star 2$ & hat \\
\hline & $2 \star 24$ & $"$ \\
\hline \multirow[t]{7}{*}{$-\frac{1}{6}$} & $\begin{array}{l}\star 28(24), \star 29(18), \star 2(10)(15), \star 2(12)(12), \star 34(12), \\
\quad \star 366, \star 446\end{array}$ & coxeter \\
\hline & $\star 2226, \star 2233, \star 2323$ & $"$ \\
\hline & $\begin{array}{l}24(12), 266,336,344,23 \infty \\
2223\end{array}$ & $\begin{array}{l}\text { stellate } \\
"\end{array}$ \\
\hline & $23 \star$ & hat \\
\hline & $4 \star 6,6 \star 3$ & $"$ \\
\hline & $2 \star 26,2 \star 33,3 \star 22$ & $"$ \\
\hline & $23 \times$ & projective \\
\hline & $\vdots$ & $\vdots$ \\
\hline \multirow[t]{12}{*}{$-\frac{1}{4}$} & $\begin{array}{l}\star 38(24), \star 39(18), \star 3(10)(15), \star 3(12)(12), \star 45(20), \\
\quad \star 46(12), \star 488, \star 55(10), \star 666,\end{array}$ & coxeter \\
\hline & $\star 2236, \star 2244, \star 2333, \star 2424, \star 2623, \star 222 \infty$ & $"$ \\
\hline & $\star 2^{5}$ & $"$ \\
\hline & $\begin{array}{l}25(20), 26(12), 288,33(12), 346,444, \\
2224\end{array}$ & $\begin{array}{l}\text { stellate } \\
"\end{array}$ \\
\hline & $24 \star$ & hat \\
\hline & $5 \star(10), 6 \star 6,8 \star 4,(12) \star 3$ & $"$ \\
\hline & $2 \star 36,2 \star 44,3 \star 23,4 \star 22$ & $"$ \\
\hline & $2 \star 36,4 \star 22,22 \star 2,24 \star$ & $"$ \\
\hline & $2 \star 222$ & $"$ \\
\hline & $24 \times$ & projective \\
\hline & $\star 2 \times$ & möbius \\
\hline & $\star \star 2$ & annular \\
\hline & $\vdots$ & $\vdots$ \\
\hline \multirow[t]{14}{*}{$-\frac{1}{2}$} & $\begin{array}{l}\star 238(24), \star 239(18), \star 23(10)(15), \star 23(12)(12), \\
\quad \star 23(15)(10), \star 23(18) 9, \star 23(24) 8, \star 245(20), \\
\quad \star 246(12), \star 2488\end{array}$ & coxeter \\
\hline & $\begin{array}{l}\star 24(12) 6, \star 24(20) 5, \star 254(20), \star 255(10), \star 25(10) 5, \\
\quad \star 264(12), \star 2666, \star 283(24), \star 2848, \star 293(18),\end{array}$ & " \\
\hline & $\begin{array}{l}\star 2(10) 3(15), \star 2(12) 3(12), \star 334(12), \star 3366, \\
\quad \star 343(12), \star 3446, \star 3464, \star 3636, \star 4444, \star 6363,\end{array}$ & " \\
\hline & $\star 22236, \star 22244, \star 22326, \star 22333, \star 22424, \star 23233$ & $"$ \\
\hline & $\star 2^{6}$ & $"$ \\
\hline & $\begin{array}{l}38(24), 39(18), 3(10)(15), 3(12)(12), \\
45(20), 46(12), 488,55(10), 666\end{array}$ & stellate \\
\hline & $2236,2244,2333,222 \infty$ & $"$ \\
\hline & $2^{5}$ & $"$ \\
\hline & $\infty \star \infty$ & hat \\
\hline & $44 \star$ & $"$ \\
\hline & $222 \star$ & $"$ \\
\hline & $\begin{array}{l}3 \star 4(12), 3 \star 66,4 \star 36,4 \star 44 \\
5 \star 2(10), 6 \star 26,6 \star 33,8 \star 24,(12) \star 23\end{array}$ & " \\
\hline & $2 \star 236,2 \star 244,2 \star 333,3 \star 223,4 \star 222$ & $"$ \\
\hline & $2 \star 2222$ & $"$ \\
\hline
\end{tabular}

Table 6 (continued)

\begin{tabular}{ll}
\hline$\chi \quad$ Orbifold & Class \\
\hline $22 \star 22$ & $"$ \\
$44 \times, 222 \times$ & projective \\
$\star 22 \times, 2 \star \times$ & möbius \\
$\star \star 22, \star 2 \star 2,2 \star \star$ & annular \\
$\circ 2$ & toroidal \\
\hline
\end{tabular}

(Robins et al., 2004a). Intermediate symmetries are manifested in lower-symmetry variants of the cubic $P, D$ and Gyroid surfaces, viz. the $t P, t D, r P D$ etc. surfaces (Fogden \& Hyde, 1992). The intermediate hyperbolic groups are therefore also crystallographic hyperbolic groups, giving a total of 79 distinct orbifolds and 131 groups. As these are all derived from $\star 246$ - the most symmetric known crystallographic hyperbolic orbifold - they are particularly important examples of crystallographic two-dimensional orbifolds. Those examples are collected by orbifold class in Table 7 .

Evidently, many more hyperbolic orbifolds are crystallographic. Further examples are derived from other 3-periodic hyperbolic surfaces, particularly TPMSs. For example, the remaining genus-three TPMSs - whose full set of translations also give an orbifold $\circ \circ \circ$ (Robins et al., 2004b) - viz. the $H$ (exagonal) surface of intrinsic symmetry $\star 2226$ and tetragonal CLP surface $(\star 2224)$ furnish 11 additional crystallographic hyperbolic orbifolds. Further examples can be deduced from larger (non-primitive) unit cells of these and other 3-periodic minimal surfaces (supergroups of $\circ^{4}$ etc.). For example, the $I$-WP surface, a genus-four cubic TPMS $(2 \star 24)$ leads to orbifolds of larger characteristic, up to $\circ^{4}$. These additional crystallographic orbifolds not listed in Table 7, are collected in Table 8 .

\section{From orbifolds to space groups}

The orbifold concept has been extended to three and higher dimensions. Systematic enumeration of crystallographic orbifolds of flat three-dimensional space $\left(\mathbb{E}^{3}\right)$ allows an alternative enumeration of the 230 euclidean space groups (Conway et al., 2001). However, the addition of a third dimension requires the introduction of an additional construction ('fibrifolds') that in our view limit the effectiveness of this approach to general users. However, the power of the orbifold concept remains a strong attraction. We recall a discussion with John Conway a few years ago on this topic, where Conway enthusiastically endorsed the simplicity of the approach. To test this, we presented Conway with an idealized model of the analcime (a zeolite) framework, and asked Conway to identify the space group. Within a couple of minutes he had identified the correct symmetry for the model $\left(\operatorname{Ia} \overline{3} d\right.$, or $8^{\circ} / 4$ in Conway's threedimensional orbifold naming system.) Clearly, the threedimensional orbifold schema is a practical possibility!

There is an alternative route to space groups, using crystallographic hyperbolic orbifolds mapped onto TPMSs embedded in $\mathbb{E}^{3}$. TPMSs in $\mathbb{E}^{3}$ offer an explicit embedding for $\mathbb{H}^{2}$ into $\mathbb{E}^{3}$, so that we can find spatial symmetries in $\mathbb{E}^{3}$ 
Table 7

Crystallographic hyperbolic groups deduced from the three-periodic $P D G$ family of minimal surfaces.

All the examples are sub (super)groups of $\star 246(\circ \circ \circ)$. (For ease of reading, we have rewritten orbifold words with multiple entries of the same character using superscripts, e.g. $\star 22222=\star 2^{5}$.)

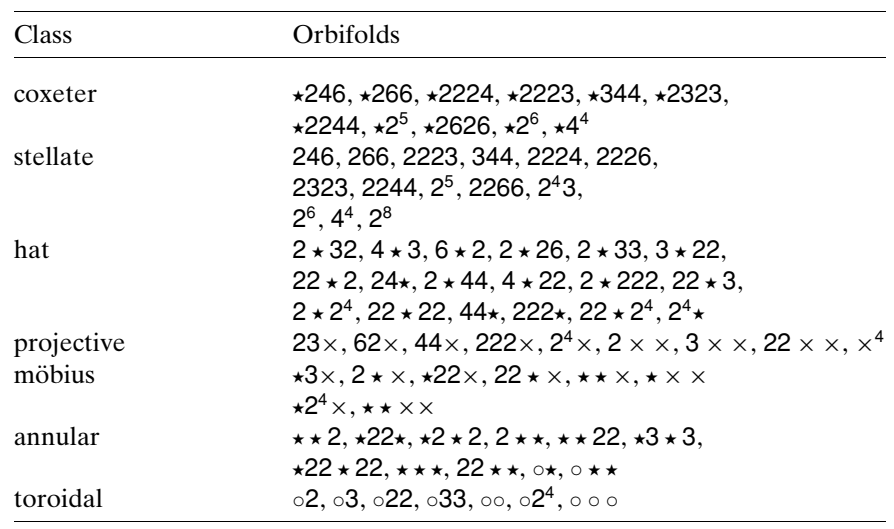

\section{Table 8}

Crystallographic hyperbolic groups deduced from the three-periodic $H$, $C L P$ and $I-W P$ minimal surfaces.

Parenthesized orbifolds are common to Table 7.

\begin{tabular}{|c|c|c|}
\hline Class & Surface & Orbifolds \\
\hline coxeter & $\begin{array}{l}H \\
C L P \\
I-W P\end{array}$ & $\begin{array}{l}\star 2226, \star 2^{4} 3, \star 2266 \\
\left(\star 2224, \star 2^{5}, \star 2244, \star 2^{6}, \star 4^{4},\right) \star 2^{8}\end{array}$ \\
\hline stellate & $\begin{array}{l}H \\
C L P \\
I-W P\end{array}$ & $\begin{array}{l}\text { (2226), 2626, }\left(2^{4} 3,2^{6}, 2^{8}\right) ; \\
(2224,) 2424,\left(2244,2^{5}, 2^{6}, 4^{4}, 2^{8}\right) ; \\
(2224,) 22422,\left(2^{6},\right) 2^{7}\end{array}$ \\
\hline hat & $\begin{array}{l}H \\
C L P\end{array}$ & $\begin{array}{l}62 \star,\left(22 \star 3,2 \star 2^{4}, 22 \star 2^{4}, 2^{4} \star\right) \\
(22 \star 2,24 \star, 2 \star 222,4 \star 22,2 \star 44,) \\
\left(222 \star, 22 \star 22,2 \star 2^{4}, 44 \star, 2^{4} \star\right)\end{array}$ \\
\hline projective & $\begin{array}{l}I-W P \\
H \\
C L P \\
I-W P\end{array}$ & $\begin{array}{l}2 \star 24,2 \star 422,(222 \star,) 22 \star 222,222 \star 2 \\
\left(3 \times \times, \times^{4}\right) ; \\
\left(222 \times, 44 \times, 2^{4} \times, 2 \times \times,\right) \times \times \times,\left(22 \times \times, \times^{4}\right) \\
\times \times \times, 2 \times \times \times, x^{5}\end{array}$ \\
\hline möbius & $\begin{array}{l}H \\
C L P \\
I-W P\end{array}$ & $\begin{array}{l}(22 \star x, \star 24 \times, \star \star x, \star \star x \times) \\
(2 \star x, \star 22 \times, \star \times \times, \star \star x) \\
\star 2 \times, 4 \star x, 2 \star 2 \times, \star 44 \times, \star 222 \times, \\
222 \star \times, 22 \star 22 \times, \star 22 \times \times, 2 \star \times \times, \star x^{4}\end{array}$ \\
\hline annular & $\begin{array}{l}H \\
C L P\end{array}$ & $\begin{array}{l}\star 3 \star, \star 33 \star, 3 \star \star,(\star 3 \star 3, \circ \star \star) \\
(\star \star 2, \star 22 \star, 2 \star \star, \star 2 \star 2, \circ \star, 22 \star \star,) \\
(\star \star \star,) \star 2^{4} \star,(\star 22 \star 22,) \circ \star \star, \star^{4}\end{array}$ \\
\hline toroidal & $\begin{array}{l}I-W P \\
H \\
C L P \\
I-W P\end{array}$ & $\begin{array}{l}(\circ \star), \circ \star 22 \\
(\circ 3, \circ 33, \circ \circ, \circ \circ \circ) \\
\left(\circ 2, \circ 22, \circ 2^{4}, \circ \circ, \circ \circ \circ\right) ; \\
(\circ 2,) \circ 44, \circ 222,(\circ \circ) \circ 2^{6}, \circ \circ 22, \circ^{4}\end{array}$ \\
\hline
\end{tabular}

induced by an orbifold-TPMS couple. Essentially, we map the two-dimensional hyperbolic symmetry group into the TPMS, forming a fundamental domain in $\mathbb{E}^{3}$. In general, there may be distinct groups of isometries with the same orbifold, so the couple refers to the specific TPMS-compatible group rather than its orbifold. Distinct groups for the same orbifold can arise due to multiple embeddings of the asymmetric domain (the orbifold) in the surface. Details are explained further in Appendix $A$. The relevant isometries for the $D$, Gyroid and $P$ surfaces are listed in Tables 9 and 10.
Table 9

Isometries for an arbitrary point $\{x, y, z\}$ in $\mathbb{E}^{3}$ due to hyperbolic reflections $r_{1}, r_{2}, r_{3}$ corresponding to the three boundary edges of the orbifold $\star 246$ mapped onto the $D$ and $P$ surfaces.

These reflections are along mirror lines running between $\star 2-\star 6, \star 2-\star 4$ and $\star 4-\star 6$ corners of the orbifold, respectively.

\begin{tabular}{llll}
\hline Surface & $r_{1}$ & $r_{2}$ & $r_{3}$ \\
\hline$D$ & $\left\{\frac{1}{4}-z, \frac{1}{4}-y, \frac{1}{4}-x\right\}$ & $\left\{\frac{1}{2}-x, y,-z\right\}$ & $\{y, x, z\}$ \\
$P$ & $\left\{\frac{1}{2}-z, y, \frac{1}{2}-x\right\}$ & $\{x,-y, z\}$ & $\left\{\frac{1}{2}-y, \frac{1}{2}-x, \frac{1}{2}-z\right\}$ \\
\hline
\end{tabular}

\section{Table 10}

Isometries for an arbitrary point $\{x, y, z\}$ in $\mathbb{E}^{3}$ due to 2-, 3- and 6-fold hyperbolic rotations $R_{1}, R_{2}, R_{3}$ of the 246 orbifold.

These operations correspond to three-dimensional isometries on the Gyroid, namely twofold rotations and $\overline{6}$ and $\overline{4}$ roto-reflections, respectively.

\begin{tabular}{lll}
\hline$R_{1}$ & $R_{2}$ & $R_{3}$ \\
\hline$\left\{\frac{1}{4}-z, \frac{1}{4}-y, \frac{1}{4}-x\right\}$ & $\left\{z, \frac{1}{2}-x,-\frac{1}{2}+y\right\}$ & $\left\{\frac{3}{4}-y, \frac{3}{4}+x, \frac{1}{4}-z\right\}$ \\
\hline
\end{tabular}

The resulting list of space groups induced by groups on the $P, D$ and Gyroid surfaces [numbered according to the schema presented in Robins et al. (2004a)] is given in Tables 11 and 12, and demonstrate the rich spectrum of space groups that result from various symmetric decorations of TPMSs. [Distinct groups are labelled by group No., corresponding to the group listing published previously (Robins et al., 2004a,b).]

We also list all space groups formed by embeddings of hyperbolic orbifolds on the two remaining genus-three TPMSs, the $H$ and $C L P$ surfaces, as well as the genus-four $I$-WP TPMS. Details of those calculations are provided in Appendix $A$. The results are listed in Tables 13 to 20 .

Systematic enumeration of space groups for generic hyperbolic surfaces is an unbounded problem. In principle, we can use this technique to generate space groups for any hyperbolic surface embedded in $\mathbb{E}^{3}$ with three independent lattice vectors, since the universal cover of such a surface is $\mathbb{H}^{2}$, and 3-periodicity of the embedding guarantees crystallographic (3-periodic) symmetry in $\mathbb{E}^{3}$. In particular, the adoption of minimal surfaces as a substrate for the hyperbolic group embedding in $\mathbb{E}^{3}$ is a convenience, rather than a necessity. Thus, for example, the hyperbolic group-spacegroup coupling is valid for decorations of 3-periodic surfaces that are parallel to the TPMS, or constant mean-curvature companions (provided the hyperbolic group excludes operations that swap sides of the surface). The minimal surfaces we have explored here are maximally symmetric embeddings of 3periodic hyperbolic surfaces with a given topology per unit cell and therefore afford the richest substrates for symmetric decorations.

\section{Conclusions}

While we find this approach to two-dimensional symmetries very intuitive, we appreciate that crystallographers who have survived thus far with other notation systems (Schönflies, 
Table 11

Correspondence between two-dimensional orbifolds and threedimensional euclidean space groups on the $P, D$ and $G$ surfaces.

(i) Simply connected orbifolds (see Table 12 for fuller caption).

\begin{tabular}{|c|c|c|c|c|c|}
\hline Class & $\begin{array}{l}\text { Subgroup } \\
\text { orbifold }\end{array}$ & $\begin{array}{l}\text { Group } \\
\text { No. }\end{array}$ & $P$ tiling & $D$ tiling & $G$ tiling \\
\hline \multirow[t]{14}{*}{ coxeter } & $\star 246$ & 131 & $\operatorname{Im} \overline{3} m$ & $P n \overline{3} m$ & (130) \\
\hline & $\star 266$ & 127 & $P n \overline{3} m$ & $F d \overline{3} m$ & (120) \\
\hline & $\star 344$ & 125 & $\operatorname{Pm} \overline{3} n$ & $P \overline{4} 3 m$ & (116) \\
\hline & $\star 2223$ & 124 & $P m \overline{3} m$ & $P 4,32$ & (118) \\
\hline & $\star 2224$ & 123 & $I 4 / \mathrm{mmm}$ & $P 4_{2} / n n m$ & (114) \\
\hline & $\star 2323$ & 119 & $\mathrm{P}_{2} 32$ & $F 43 m$ & (92) \\
\hline & $\star 2244 a$ & 107 & $P 4_{2} / m m c$ & $P \overline{4} 2 m$ & (86) \\
\hline & $\star 2244 b$ & 103 & $\mathrm{P} 4 / \mathrm{nmm}$ & $I 4_{1} /$ amd & (87) \\
\hline & $\star 2^{5} a$ & 102 & $P 4 / m m m$ & $\mathrm{P}_{2} 22$ & (77) \\
\hline & $\star 2^{5} b$ & 101 & Fmmm & Cтma & (76) \\
\hline & $\star 2626$ & 96 & $R \overline{3} m$ & $R \overline{3} m$ & (50) \\
\hline & $\star 4444$ & 64 & $P \overline{4} m 2$ & $I \overline{4} m 2$ & (17) \\
\hline & $\star 2^{6} a$ & 83 & Cтmma & Imma & (27) \\
\hline & $\star 2^{6} \mathrm{~b}$ & 55 & Pmmm & $P 222$ & (14) \\
\hline \multirow[t]{21}{*}{ stellate } & 246 & 130 & $P n \overline{3} n$ & $F d \overline{3} c$ & $I a \overline{3} d$ \\
\hline & 266 & 120 & $\operatorname{Pn} \overline{3}$ & $F d \overline{3}$ & $I a \overline{3}$ \\
\hline & 2223 & 118 & $P 432$ & $F 4_{1} 32$ & $I 4_{1} 32$ \\
\hline & 344 & 116 & $P \overline{4} 3 n$ & $F \overline{4} 3 c$ & $I \overline{4} 3 d$ \\
\hline & 2224 & 114 & $P 4 / n n c$ & $I 4_{1} /$ acd & $I 4_{1} / a c d$ \\
\hline & 2226 & 93 & $R \overline{3} c$ & $R \frac{1}{3} c$ & $R \frac{1}{3} c$ \\
\hline & 2233 & 92 & $P 23$ & $F 23$ & $I 2,3$ \\
\hline & 2244 & 87 & $P 4 / n$ & $I 4_{1} / a$ & $I 4_{1} / a$ \\
\hline & 2244 & 86 & $P \overline{4} 2 c$ & $I \overline{4} c 2$ & $I \overline{4} 2 d$ \\
\hline & 2244 & 78 & $P \overline{4} n 2$ & $I \overline{4} 2 d$ & $I \overline{4} c 2$ \\
\hline & $2^{5}$ & 77 & $P 422$ & $I 4_{1} 22$ & $I 4_{1} 22$ \\
\hline & $2^{5}$ & 76 & Ccca & $I b c a$ & Fddd \\
\hline & $2^{5}$ & 54 & Pnnn & Fddd & $I b c a$ \\
\hline & 2266 & 50 & $R \overline{3}$ & $R \overline{3}$ & $R \overline{3}$ \\
\hline & $2^{4} 3$ & 49 & $R 32$ & $R 32$ & $R 32$ \\
\hline & $2^{6}$ & 44 & $C 2 / c$ & $C 2 / c$ & $C 2 / c$ \\
\hline & $2^{6}$ & 27 & $P 2 / c$ & $C 2 / c$ & $C 2 / c$ \\
\hline & $2^{6}$ & 16 & C222 & $I 212_{1} 2_{1}$ & $F 222$ \\
\hline & $2^{6}$ & 14 & $P 222$ & $F 222$ & $I 2_{1} 2_{1} 2_{1}$ \\
\hline & $4^{4}$ & 17 & $P \overline{4}$ & $I \overline{4}$ & $I \overline{4}^{-1}$ \\
\hline & $2^{8}$ & 2 & $P \overline{1}$ & $P \overline{1}$ & $P \overline{1}$ \\
\hline \multirow[t]{29}{*}{ hat } & $2 \star 32$ & 129 & $I 432$ & $F d \overline{3} m$ & (118) \\
\hline & $4 \star 3$ & 128 & $I \overline{4} 3 m$ & $F d \overline{3} c$ & (116) \\
\hline & $6 \star 2$ & 126 & $\operatorname{Im} \overline{3}$ & $P n \overline{3}$ & (120) \\
\hline & $2 \star 26$ & 122 & $R \overline{3} m$ & $R \overline{3} m$ & (93) \\
\hline & $2 \star 33$ & 117 & $P \overline{4} 3 m$ & $F 4_{1} 32$ & (92) \\
\hline & $3 \star 22$ & 115 & $P m \overline{3}$ & $P 23$ & (92) \\
\hline & $22 \star 2$ & 113 & $I 422$ & $I 4_{1} /$ amd & (77) \\
\hline & $22 \star 2$ & 111 & $\mathrm{P}_{2} / n m c$ & $I 4_{1} / a c d$ & (76) \\
\hline & $24 \star$ & 110 & $I \overline{4} 2 \mathrm{~m}$ & $I 4_{1} /$ acd & (86) \\
\hline & $24 \star$ & 109 & $I 4 / m$ & $P 4_{2} / n$ & (87) \\
\hline & $2 \star 44$ & 108 & $I \overline{4} m 2$ & $I 4_{1} /$ amd & (78) \\
\hline & $4 \star 22$ & 106 & $\mathrm{P}_{2} / \mathrm{mnm}$ & $P \overline{4} n 2$ & (78) \\
\hline & $2 \star 222$ & 104 & $\mathrm{P}_{2} / \mathrm{nnm}$ & $I 4_{1} /$ amd & (54) \\
\hline & $2 \star 222$ & 100 & Immm & Pnnn & (54) \\
\hline & $22 \star 3$ & 99 & $R 32$ & $R \overline{3} m$ & (49) \\
\hline & $22 \star 3$ & 97 & $R \overline{3} m$ & $R 32$ & (49) \\
\hline & $2 \star 2^{4}$ & 90 & $\mathrm{Cmcm}$ & Ibam & (44) \\
\hline & $2 \star 2^{4}$ & 57 & $\mathrm{Cmmm}$ & $C 222$ & (16) \\
\hline & $22 \star 22$ & 89 & $C 2 / m$ & $C 2 / m$ & (44) \\
\hline & $22 \star 22$ & 81 & $\mathrm{P}_{2} 22$ & $I \overline{4} m 2$ & (14) \\
\hline & $22 \star 22$ & 75 & $F 222$ & Imma & (16) \\
\hline & $22 \star 22$ & 67 & Pmmn & $F d d d$ & (27) \\
\hline & $22 \star 22$ & 66 & $P \overline{4} m 2$ & $I 4_{1} 22$ & (16) \\
\hline & $44 \star$ & 74 & $P 4_{2} / m$ & $P \overline{4}$ & (17) \\
\hline & $44 \star$ & 62 & $P \overline{4} 2_{1} m$ & $I \overline{4} 2 d$ & (17) \\
\hline & $222 \star$ & 71 & $C 2 / m$ & $P 2 / c$ & (27) \\
\hline & $222 \star$ & 65 & $P \overline{4} 2 m$ & $I 4_{1} 22$ & (14) \\
\hline & $22 \star 2^{4}$ & 36 & $C 2 / m$ & $C 2 / m$ & (2) \\
\hline & $2^{4} \star$ & 23 & $P 2_{1} / m$ & $C 2 / c$ & (2) \\
\hline
\end{tabular}

Table 12

Correspondence between two-dimensional orbifolds and threedimensional euclidean space groups on the $P, D$, and $G$ surfaces.

(ii) Multiply connected orbifolds. The $P, D, G$ tiling columns list the euclidean space groups of the surface reticulations that arise from each hyperbolic symmetry. Space groups on the Gyroid are listed for stellate and toroidal orbifolds; these three-dimensional groups apply also to all supergroups realised by adding mirrors and/or cross-caps. If the two-dimensional group is a supergroup of a stellate or toroidal group, the Gyroid space group is determined by finding the root stellate or toroidal group (listed by twodimensional group No. in the Gyroid column), then checking the space group for the root group.

\begin{tabular}{|c|c|c|c|c|c|}
\hline Class & $\begin{array}{l}\text { Subgroup } \\
\text { orbifold }\end{array}$ & $\begin{array}{l}\text { Group } \\
\text { No. }\end{array}$ & $P$ tiling & $D$ tiling & $G$ tiling \\
\hline \multirow[t]{19}{*}{ projective } & $23 x$ & 121 & $I 23$ & $F d \overline{3}$ & (92) \\
\hline & $62 x$ & 94 & $R \overline{3}$ & $R \overline{3}$ & (50) \\
\hline & $44 \times$ & 84 & $I \overline{4}$ & $I 4_{1} / a$ & (17) \\
\hline & $2^{3} x$ & 79 & $P 422_{1} 2$ & $I \overline{4} 2 d$ & (16) \\
\hline & $2^{3} x$ & 58 & $P 4_{2} / n$ & $I 4_{1} / a$ & (27) \\
\hline & $2^{3} x$ & 53 & $I 222$ & Fddd & (14) \\
\hline & $2^{4} x$ & 15 & $P \overline{1}$ & $P \overline{1}$ & (2) \\
\hline & $2 \times x$ & 85 & $P \overline{4} 2_{1} c$ & $I 4_{1} c d$ & (16) \\
\hline & $2 \times x$ & 56 & $I 4$ & $I 4_{1} / a$ & (18) \\
\hline & $3 \times \times$ & 52 & $R 3$ & $R R^{\overline{3}}$ & (13) \\
\hline & $3 \times x$ & 46 & $R \overline{3}$ & $R 3$ & (13) \\
\hline & $22 \times x$ & 32 & $C 2$ & $C 2 / c$ & (7) \\
\hline & $22 \times x$ & 29 & $P 2_{1} 2_{1} 2$ & $F d d 2$ & (7) \\
\hline & $22 \times x$ & 26 & $\mathrm{P4}_{2}$ & $I \overline{4}$ & (7) \\
\hline & $22 \times x$ & 19 & $P \overline{4}^{2}$ & $I 4_{1}$ & (7) \\
\hline & $x^{3}$ & 31 & $P 2_{1} / c$ & $P c$ & (8) \\
\hline & $x^{4}$ & 5 & $P 2_{1}$ & $C c$ & (1) \\
\hline & $x^{4}$ & 4 & $P 1$ & $P \overline{1}$ & (1) \\
\hline & $x^{4}$ & 3 & $P \overline{1}$ & $P 1$ & (1) \\
\hline
\end{tabular}

möbiu

$\star 3 \times$

$\star 3 \times$

$2 \star x$

$2 \star x$

$\star 22 \times$

$\star 22 \times$

$22 \star x$

$22 \star x$

$22 \star x$

$\star \star x$

$\star \star x$

$\star x \times$

$\star x \times$

$\star x \times$

$\star 2^{4} \times$

$\star \star x \times$

$\star \star x \times$

$\star \star 2$

$\star \star 2$

$\star \star 22$

$\star \star 22$

$\star \star 22$

$\star 2 \star 2$

$\star 2 \star 2$

$2 \star \star$

$2 \star \star$

$2 \star \star$

$\star 3 \star 3$

$\star 3 \star 3$

$\star 22 \star 22$

$\star 22 \star 22$

$\star \star \star$

$22 \star \star$

$22 \star \star$

$0 \star$

$\circ \star \star$

\begin{tabular}{l}
98 \\
95 \\
72 \\
63 \\
82 \\
69 \\
42 \\
37 \\
35 \\
39 \\
33 \\
45 \\
41 \\
40 \\
34 \\
10 \\
9 \\
112 \\
105 \\
91 \\
70 \\
60 \\
80 \\
68 \\
88 \\
73 \\
59 \\
47 \\
48 \\
28 \\
25 \\
38 \\
22 \\
20 \\
30 \\
6 \\
\hline
\end{tabular}

$R 3 m \quad R 3 c$

Pnnm Pnn2

$\mathrm{P}_{2} \mathrm{~nm} \quad \mathrm{I} \overline{4} 2 \mathrm{~d}$

$P \overline{4} n 2 \quad I 4{ }_{1} m d$

Imm2 Fddd (24)

$C 2 \quad C 2 / m \quad(11)$

$C 2 / m \quad C 2$

$C 222_{1} \quad$ Ima2 (11)

Ama2 Ima2 (12)

$\mathrm{Cmc2} 2_{1} \quad \mathrm{Iba2} \quad$ (12)

$\mathrm{C} 2 / \mathrm{c} \quad \mathrm{Cm}$

$\mathrm{Pmn}_{1} \quad \mathrm{Fdd} 2$

$\mathrm{Cm} \quad \mathrm{C} 2 / \mathrm{c}$

Amm2 I222 (11)

$\mathrm{C2} \quad \mathrm{Cm}$

$\mathrm{Cm} \quad \mathrm{C2}$

$\mathrm{P} 4 / \mathrm{mnc} \quad \mathrm{P}_{2} \mathrm{~nm} \quad(61)$

$I 4 \mathrm{~mm} \quad I 4_{1} /$ acd

Fmm2 Ibam (43)

Cccm Cmm2 (21)

$\mathrm{P} 4 \mathrm{~mm} \quad \mathrm{I}_{1} 22$

$\mathrm{P}_{2} 12 \quad I 4_{1} m d$

$\mathrm{P}_{2} \mathrm{mc} \quad \mathrm{I} \overline{4} \mathrm{c} 2$

Cmca Abm2 (43)

$\mathrm{P4} / \mathrm{m} \quad \mathrm{P}_{2}$

Fmm2 Ibca (21)

Fdd2 R32 (13)

$R 32 \quad R 3 m$

C222 Imm2

Pmm2 F222 (7)

Abm2 Ima2 (8)

$P 2 / m \quad P 2$

Cmm2 I2 $22_{1} 2_{1} \quad(7)$

$\mathrm{Cm} \quad \mathrm{C} 2 / \mathrm{c}$

$\mathrm{Pm} \quad \mathrm{C2}$ 
Table 12 (continued)

\begin{tabular}{llllll}
\hline \multirow{2}{*}{ Class } & $\begin{array}{l}\text { Subgroup } \\
\text { orbifold }\end{array}$ & $\begin{array}{l}\text { Group } \\
\text { No. }\end{array}$ & $P$ tiling & $D$ tiling & $G$ tiling \\
\hline \multirow{2}{*}{ toroidal } & $\circ 2$ & 61 & $P 4 n c$ & $I 4_{1} c d$ & $I 4_{1} c d$ \\
& $\circ 3$ & 51 & $R 3 c$ & $R 3 c$ & $R 3 c$ \\
& $\circ 22$ & 43 & $A b a 2$ & $I b a 2$ & $F d d 2$ \\
& $\circ 22$ & 24 & $P n n 2$ & $F d d 2$ & $I b a 2$ \\
& $\circ 22$ & 21 & $C c c 2$ & $I b a 2$ & $F d d 2$ \\
& $\circ 22$ & 18 & $P 4$ & $I 4_{1}$ & $I 4_{1}$ \\
& $\circ 33$ & 13 & $R 3$ & $R 3$ & $R 3$ \\
& $\circ$ & 12 & $C c$ & $C c$ & $C c$ \\
& $\circ$ & 8 & $P c$ & $C c$ & $C c$ \\
& $\circ 2^{4}$ & 11 & $C 2$ & $C 2$ & $C 2$ \\
& $\circ 2^{4}$ & 7 & $P 2$ & $C 2$ & $C 2$ \\
& $\circ \circ$ & 1 & $P 1$ & $P 1$ & $P 1$ \\
\hline
\end{tabular}

\section{Table 13}

Isometries in three-dimensional space on the CLP surface for an arbitrary point $\{x, y, z\}$ in $\mathbb{E}^{3}$ due to hyperbolic reflections $r_{1}, r_{2}, r_{3}, r_{4}$ corresponding to edges of the $\star 2224$ of the CLP surface (Fig. 8), and isometries $R_{1}, R_{2}, R_{3}$ and $R_{4}$ in Robins et al. (2004b) on the $H$ surface (oriented $P \overline{6} \mathrm{~m} 2$ setting, with $\star 6$ site at $0,0,1 / 4$ and a $\star 2$ site at $0,1 / 2,0$ ).

\begin{tabular}{lllll}
\hline Surface & $r_{1}$ & $r_{2}$ & $r_{3}$ & $r_{4}$ \\
\hline$C L P$ & $\left\{\frac{1}{2}-x, \frac{1}{2}-y, z\right\}$ & $\{x, y,-z\}$ & $\{x,-y, z\}$ & $\left\{\frac{1}{2}+y,-\frac{1}{2}+x, \frac{1}{2}-z\right\}$ \\
$H$ & $\left\{-\frac{x}{2}+\frac{\sqrt{3} y}{2}, \frac{\sqrt{3} x}{2}+\frac{y}{2}, z\right\}$ & $\left\{-x, y, \frac{1}{2}-z\right\}$ & $\{x,-y, z\}$ & $\{x, y,-z\}$ \\
\hline
\end{tabular}

Table 14

Isometries for an arbitrary point $\{x, y, z\}$ in $\mathbb{E}^{3}$ due to hyperbolic reflections $r_{1}, r_{2}$ and rotation $R_{1}$ corresponding to generators of the $2 \star 24$ orbifold mapped onto the $I$-WP surface.

\begin{tabular}{lll}
\hline$r_{1}$ & $r_{2}$ & $R_{1}$ \\
\hline$\{y, x, z\}$ & $\{x, y,-z\}$ & $\left\{\frac{1}{2}-x,-\frac{1}{2}+z, \frac{1}{2}+y\right\}$ \\
\hline
\end{tabular}

Hermann-Mauguin) will wonder why we should argue for the introduction of yet another notation system. The advantages of the orbifold paradigm are many, particularly coupled with the elegant naming scheme for orbifolds developed by Conway. A particular attraction is the ability to decipher the meaning of a symmetry group without the need to resort to look-up tables: familiarity with the topological characters in orbifold words, $\circ, \star$ and $\times$, is sufficient to decipher the symmetries encoded by an orbifold from its name alone. The addition of a further simple set of rules assigning a characteristic to every character in an orbifold word allows further explication of group order (for point groups) and groupsubgroup relations (for point and hyperbolic groups). The formula for these characteristics [equation (4)], while obscure from a conventional crystallographic viewpoint, is lucid from the perspective of topology and differential geometry. Therein lies perhaps the most substantial argument for the adoption of the orbifold concept. It offers a simple reconciliation of euclidean and non-euclidean patterns and ties questions of symmetry to the broader domain of geometry and topology. Thus, for example, all two-dimensional symmetries are collected within a single conceptual framework and the fragmentation of ad hoc naming systems for rosette or point groups, plane and frieze groups, is repaired. Lastly, and most significantly, the huge variety of hyperbolic groups can be
Table 15

Subgroups of $\star 2224$ commensurate with a unit translational cell of the $C L P$ surface.

\begin{tabular}{|c|c|c|c|c|}
\hline & Orbifold & Index & $\begin{array}{l}\text { Conjugacy } \\
\text { class size }\end{array}$ & $\begin{array}{l}\text { Subgroup } \\
\text { generators in } * 2224 / \mathrm{T}\end{array}$ \\
\hline 1 & 000 & 32 & 1 & identity \\
\hline 2 & $\times \times \times \times$ & 16 & 1 & $r_{2} r_{3} r_{4} r_{3} r_{4}$ \\
\hline 3 & $\star \star \star \star$ & 16 & 1 & $r_{1}$ \\
\hline 4 & 22222222 & 16 & 1 & $r_{1} r_{2}$ \\
\hline 5 & $\star \star \star \star$ & 16 & 1 & $r_{2}$ \\
\hline 6 & $\times \times \times \times$ & 16 & 1 & $r_{1} r_{3} r_{4} r_{3} r_{4}$ \\
\hline 7 & $\infty$ & 16 & 1 & $r_{1} r_{2} r_{3} r_{4} r_{3} r_{4}$ \\
\hline 8 & $\circ 2222$ & 16 & 1 & $r_{3} r_{4} r_{3} r_{4}$ \\
\hline 9 & $0 \star \star$ & 16 & 2 & $r_{4}$ \\
\hline 10 & $\circ 2222$ & 16 & 2 & $r_{2} r_{3}$ \\
\hline 11 & ০ & 16 & 2 & $r_{2} r_{4}$ \\
\hline 12 & ০ & 16 & 2 & $r_{1} r_{3}$ \\
\hline 13 & $\times \times \times \times$ & 16 & 2 & $r_{1} r_{2} r_{3}$ \\
\hline 14 & ०2222 & 16 & 2 & $r_{1} r_{4}$ \\
\hline 15 & $\circ \star \star$ & 16 & 2 & $r_{3}$ \\
\hline 16 & $x \times \times \times$ & 16 & 2 & $r_{1} r_{2} r_{4}$ \\
\hline 17 & 4444 & 8 & 1 & $r_{4} r_{3}$ \\
\hline 18 & $22 \times \times$ & 8 & 1 & $r_{1} r_{3} r_{4}$ \\
\hline 19 & $\circ 22$ & 8 & 1 & $r_{3} r_{1}, r_{4} r_{3} r_{4} r_{1}$ \\
\hline 20 & $\star 22 \star 22$ & 8 & 1 & $r_{3}, r_{4} r_{3} r_{4}$ \\
\hline 21 & 222222 & 8 & 1 & $r_{2} r_{1}, r_{4} r_{3} r_{4} r_{3}$ \\
\hline 22 & $2222 x$ & 8 & 1 & $r_{2} r_{1}, r_{1} r_{3} r_{4} r_{3} r_{4}$ \\
\hline 23 & $22 \times \times$ & 8 & 1 & $r_{1} r_{2} r_{3}, r_{4} r_{3} r_{4} r_{3}$ \\
\hline 24 & $\star 22222222$ & 8 & 1 & $r_{1}, r_{2}$ \\
\hline 25 & $\star \star \times$ & 8 & 1 & $r_{2}, r_{1} r_{3} r_{4} r_{3} r_{4}$ \\
\hline 26 & 222222 & 8 & 1 & $r_{3} r_{2}, r_{4} r_{3} r_{4} r_{2}$ \\
\hline 27 & $\circ 22$ & 8 & 1 & $r_{4} r_{2}, r_{3} r_{4} r_{3} r_{2}$ \\
\hline 28 & $22 \star \star$ & 8 & 1 & $r_{1}, r_{4} r_{3} r_{4} r_{3}$ \\
\hline 29 & $\star \star \times$ & 8 & 1 & $r_{1}, r_{2} r_{3} r_{4} r_{3} r_{4}$ \\
\hline 30 & $\star 22 \star 22$ & 8 & 1 & $r_{4}, r_{3} r_{4} r_{3}$ \\
\hline 31 & $22 \times \times$ & 8 & 1 & $r_{1} r_{2} r_{4}, r_{4} r_{3} r_{4} r_{3}$ \\
\hline 32 & $22 \times \times$ & 8 & 1 & $r_{2} r_{3} r_{4}$ \\
\hline 33 & $\circ 22$ & 8 & 1 & $r_{2} r_{3} r_{4} r_{1}$ \\
\hline 34 & 222222 & 8 & 1 & $r_{4} r_{1}, r_{3} r_{4} r_{3} r_{1}$ \\
\hline 35 & $22 \star \star$ & 8 & 1 & $r_{2}, r_{4} r_{3} r_{4} r_{3}$ \\
\hline 36 & $\star \star 2222$ & 8 & 2 & $r_{2}, r_{3}$ \\
\hline 37 & $\star \times \times$ & 8 & 2 & $r_{3}, r_{1} r_{2} r_{4} r_{3} r_{4}$ \\
\hline 38 & $\star \star \star$ & 8 & 2 & $r_{1}, r_{3}$ \\
\hline 39 & $22 \star \star$ & 8 & 2 & $r_{3}, r_{4} r_{3} r_{4} r_{2}$ \\
\hline 40 & $\star \star \star$ & 8 & 2 & $r_{1}, r_{4} r_{2}$ \\
\hline 41 & $2222 \star$ & 8 & 2 & $r_{2} r_{1}, r_{4}$ \\
\hline 42 & $O \star$ & 8 & 2 & $r_{3}, r_{4} r_{3} r_{4} r_{1}$ \\
\hline 43 & $\star \star 2222$ & 8 & 2 & $r_{1}, r_{4}$ \\
\hline 44 & 222222 & 8 & 2 & $r_{2} r_{1}, r_{3} r_{1}$ \\
\hline 5 & $2222 \star$ & 8 & 2 & $r_{2} r_{1}, r_{3}$ \\
\hline 46 & $22 \star \star$ & 8 & 2 & $r_{1}, r_{3} r_{2}$ \\
\hline 47 & $\star \star \star$ & 8 & 2 & $r_{2}, r_{3} r_{1}$ \\
\hline 48 & $\star \star \star$ & 8 & 2 & $r_{2}, r_{4}$ \\
\hline 49 & $22 \star \star$ & 8 & 2 & $r_{2}, r_{4} r_{1}$ \\
\hline 50 & $\circ 22$ & 8 & 2 & $r_{3} r_{1}, r_{4} r_{3} r_{4} r_{2}$ \\
\hline 51 & $\times \times \times$ & 8 & 2 & $r_{3} r_{1}, r_{1} r_{2} r_{4} r_{3} r_{4}$ \\
\hline 52 & $22 \times \times$ & 8 & 2 & $r_{4} r_{1}, r_{1} r_{2} r_{3} r_{4} r_{3}$ \\
\hline 53 & $22 \times \times$ & 8 & 2 & $r_{3} r_{2}, r_{1} r_{2} r_{4} r_{3} r_{4}$ \\
\hline 54 & $x \times x$ & 8 & 2 & $r_{4} r_{2}, r_{1} r_{2} r_{3} r_{4} r_{3}$ \\
\hline 55 & $\circ 22$ & 8 & 2 & $r_{4} r_{1}, r_{3} r_{4} r_{3} r_{2}$ \\
\hline 56 & $22 \star \star$ & 8 & 2 & $r_{4}, r_{3} r_{4} r_{3} r_{1}$ \\
\hline 57 & $0 \star$ & 8 & 2 & $r_{4}, r_{3} r_{4} r_{3} r_{2}$ \\
\hline 58 & $\star \times \times$ & 8 & 2 & $r_{4}, r_{1} r_{2} r_{3} r_{4} r_{3}$ \\
\hline 59 & 222222 & 8 & 2 & $r_{2} r_{1}, r_{4} r_{1}$ \\
\hline 60 & $222 \star$ & 4 & 1 & $r_{1}, r_{3} r_{2}, r_{4} r_{3} r_{4} r_{2}$ \\
\hline 61 & $\star \star 22$ & 4 & 1 & $r_{1}, r_{3}, r_{4} r_{3} r_{4}$ \\
\hline 62 & $44 \star$ & 4 & 1 & $r_{2}, r_{4} r_{3}$ \\
\hline 63 & $\star 222222$ & 4 & 1 & $r_{2}, r_{3}, r_{4} r_{3} r_{4}$ \\
\hline 64 & $44 \star$ & 4 & 1 & $r_{1}, r_{4} r_{3}$ \\
\hline 65 & $22 \star 22$ & 4 & 1 & $r_{2} r_{1}, r_{3}, r_{4} r_{3} r_{4}$ \\
\hline 66 & $2 \star \star$ & 4 & 1 & $r_{2}, r_{3} r_{1}, r_{4} r_{3} r_{4} r_{1}$ \\
\hline 67 & $\star 4444$ & 4 & 1 & $r_{3}, r_{4}$ \\
\hline 68 & $22 \star 22$ & 4 & 1 & $r_{3}, r_{4} r_{1}$ \\
\hline 69 & $\star 2 \star 2$ & 4 & 1 & $r_{3}, r_{4} r_{2}$ \\
\hline
\end{tabular}


$\underline{\text { Table } 15 \text { (continued) }}$

\begin{tabular}{|c|c|c|c|c|}
\hline & Orbifold & Index & $\begin{array}{l}\text { Conjugacy } \\
\text { class size }\end{array}$ & $\begin{array}{l}\text { Subgroup } \\
\text { generators in } * 2224 / T\end{array}$ \\
\hline 70 & $\star 22 \times$ & 4 & 1 & $r_{3}, r_{1} r_{2} r_{4}$ \\
\hline 71 & $2 \times \times$ & 4 & 1 & $r_{3} r_{1}, r_{1} r_{2} r_{4}$ \\
\hline 72 & $2 \star 2222$ & 4 & 1 & $r_{1}, r_{2}, r_{4} r_{3} r_{4} r_{3}$ \\
\hline 73 & $222 \times$ & 4 & 1 & $r_{2} r_{1}, r_{1} r_{3} r_{4}$ \\
\hline 74 & $\star 222222$ & 4 & 1 & $r_{1}, r_{4}, r_{3} r_{4} r_{3}$ \\
\hline 75 & $222 \times$ & 4 & 1 & $r_{4} r_{1}, r_{1} r_{2} r_{3}$ \\
\hline 76 & $2 \star \star$ & 4 & 1 & $r_{1}, r_{4} r_{2}, r_{3} r_{4} r_{3} r_{2}$ \\
\hline 77 & $22 \star 22$ & 4 & 1 & $r_{3} r_{2}, r_{4}$ \\
\hline 78 & $2 \star \times$ & 4 & 1 & $r_{1}, r_{2} r_{3} r_{4}$ \\
\hline 79 & 22222 & 4 & 1 & $r_{3} r_{2}, r_{4} r_{1}$ \\
\hline 80 & 22222 & 4 & 1 & $r_{2} r_{1}, r_{3} r_{1}, r_{4} r_{3} r_{4} r_{1}$ \\
\hline 81 & $\star 2 \star 2$ & 4 & 1 & $r_{3} r_{1}, r_{4}$ \\
\hline 82 & 2244 & 4 & 1 & $r_{3} r_{1}, r_{4} r_{1}$ \\
\hline 83 & $\circ 2$ & 4 & 1 & $r_{3} r_{1}, r_{4} r_{2}$ \\
\hline 84 & 22222 & 4 & 1 & $r_{2} r_{1}, r_{4} r_{1}, r_{3} r_{4} r_{3} r_{1}$ \\
\hline 85 & $\star \star 22$ & 4 & 1 & $r_{2}, r_{4}, r_{3} r_{4} r_{3}$ \\
\hline 86 & $22 \star 22$ & 4 & 1 & $r_{2} r_{1}, r_{4}, r_{3} r_{4} r_{3}$ \\
\hline 87 & $222 \star$ & 4 & 1 & $r_{2}, r_{4} r_{1}, r_{3} r_{4} r_{3} r_{1}$ \\
\hline 88 & $\star 22 \times$ & 4 & 1 & $r_{4}, r_{1} r_{2} r_{3}$ \\
\hline 89 & $2 \star x$ & 4 & 1 & $r_{2}, r_{1} r_{3} r_{4}$ \\
\hline 90 & 2244 & 4 & 1 & $r_{2} r_{1}, r_{4} r_{3}$ \\
\hline 91 & 2244 & 4 & 1 & $r_{3} r_{2}, r_{4} r_{2}$ \\
\hline 92 & $44 \times$ & 4 & 1 & $r_{4} r_{3}, r_{1} r_{2} r_{3}$ \\
\hline 93 & $222 \times$ & 4 & 1 & $r_{3} r_{2}, r_{1} r_{2} r_{4}$ \\
\hline 94 & $2 \times \times$ & 4 & 1 & $r_{4} r_{2}, r_{1} r_{2} r_{3}$ \\
\hline 95 & $222 \star$ & 4 & 2 & $r_{2} r_{1}, r_{4}, r_{3} r_{4} r_{3} r_{1}$ \\
\hline 96 & $2 \star \star$ & 4 & 2 & $r_{1}, r_{3}, r_{4} r_{3} r_{4} r_{2}$ \\
\hline 97 & $\star 222222$ & 4 & 2 & $r_{1}, r_{2}, r_{4}$ \\
\hline 98 & $\star 222222$ & 4 & 2 & $r_{1}, r_{2}, r_{3}$ \\
\hline 99 & $2 \star \star$ & 4 & 2 & $r_{2}, r_{4}, r_{3} r_{4} r_{3} r_{1}$ \\
\hline 100 & $222 \star$ & 4 & 2 & $r_{2} r_{1}, r_{3}, r_{4} r_{3} r_{4} r_{1}$ \\
\hline 101 & $\star \star 22$ & 4 & 2 & $r_{1}, r_{4}, r_{3} r_{4} r_{3} r_{2}$ \\
\hline 102 & $\star \star 22$ & 4 & 2 & $r_{2}, r_{3}, r_{4} r_{3} r_{4} r_{1}$ \\
\hline 103 & 22222 & 2 & 1 & $r_{1}, r_{2}, r_{3}, r_{4} r_{3} r_{4}$ \\
\hline 104 & $\star 2244$ & 2 & 1 & $r_{1}, r_{3}, r_{4}$ \\
\hline 105 & $\star \star 2$ & 2 & 1 & $r_{1}, r_{3}, r_{4} r_{2}$ \\
\hline 106 & $\star 2244$ & 2 & 1 & $r_{2}, r_{3}, r_{4}$ \\
\hline 107 & $2 \star 222$ & 2 & 1 & $r_{2}, r_{3}, r_{4} r_{1}$ \\
\hline 108 & $2 \star 44$ & 2 & 1 & $r_{2} r_{1}, r_{3}, r_{4}$ \\
\hline 109 & $22 \star 2$ & 2 & 1 & $r_{2} r_{1}, r_{3}, r_{4} r_{1}$ \\
\hline 110 & $\star 22222$ & 2 & 1 & $r_{1}, r_{2}, r_{4}, r_{3} r_{4} r_{3}$ \\
\hline 111 & $4 \star 22$ & 2 & 1 & $r_{1}, r_{2}, r_{4} r_{3}$ \\
\hline 112 & 2222 & 2 & 1 & $r_{1}, r_{3} r_{2}, r_{4}$ \\
\hline 113 & $42 \star$ & 2 & 1 & $r_{1}, r_{3} r_{2}, r_{4} r_{2}$ \\
\hline 114 & $\star \star 2$ & 2 & 1 & $r_{2}, r_{3} r_{1}, r_{4}$ \\
\hline 115 & $42 \star$ & 2 & 1 & $r_{2}, r_{3} r_{1}, r_{4} r_{1}$ \\
\hline 116 & $22 \star 2$ & 2 & 1 & $r_{2} r_{1}, r_{3} r_{1}, r_{4}$ \\
\hline 117 & 2224 & 2 & 1 & $r_{2} r_{1}, r_{3} r_{1}, r_{4} r_{1}$ \\
\hline 118 & $\star 2224$ & 1 & 1 & $r_{1}, r_{2}, r_{3}, r_{4}$ \\
\hline
\end{tabular}

explored at once, within the same conceptual framework as that adopted for better known two-dimensional discrete groups. We encourage the curious crystallographer to explore this topological approach to symmetry themselves, as familiarity with symmetry leads to deep insights into lowdimensional topology, and vice versa.

\section{APPENDIX $\boldsymbol{A}$ \\ Procedure for enumerating space groups from orbifold-TPMS mappings}

\section{A1. $P, D$ and Gyroid embeddings}

The process involves enumeration of all group words within a unit cell shared by the surfaces (strictly, the $\star 246$ group
Table 16

Correspondence between two-dimensional orbifolds and threedimensional euclidean space groups on the CLP surface: simply connected orbifolds.

\begin{tabular}{|c|c|c|c|}
\hline Class & Orbifold & Group No. & 3D space group \\
\hline \multirow{11}{*}{ coxeter } & $\star 2224$ & 118 & $\mathrm{P}_{2} / \mathrm{mcm}$ \\
\hline & $\star 2^{5}$ & 110 & Pccm \\
\hline & $\star 2244$ & 106 & $\mathrm{P}_{2} / \mathrm{mmc}$ \\
\hline & $\star 2244$ & 104 & $P \overline{4} 2 m$ \\
\hline & $\star 2^{5}$ & 103 & $\mathrm{Cmmm}$ \\
\hline & $\star 2^{6}$ & 98 & Pmna \\
\hline & $\star 2^{6}$ & 97 & Cccm \\
\hline & $\star 2^{6}$ & 74 & $P 222$ \\
\hline & $\star 4^{4}$ & 67 & $P \overline{4} m 2$ \\
\hline & $\star 2^{6}$ & 63 & Pmmm \\
\hline & $\star 2^{8}$ & 24 & $P 2 / m$ \\
\hline \multirow[t]{14}{*}{ stellate } & 2224 & 117 & $P 4_{2} / n b c$ \\
\hline & 2244 & 91 & $P \overline{4} 2 c$ \\
\hline & 2244 & 90 & $P 4_{2} / n$ \\
\hline & $2^{5}$ & 84 & Ccca \\
\hline & 2244 & 82 & $P \overline{4} b 2$ \\
\hline & $2^{5}$ & 80 & Pban \\
\hline & $2^{5}$ & 79 & $P 4_{2} 22$ \\
\hline & $2^{6}$ & 59 & $C 2 / c$ \\
\hline & $2^{6}$ & 44 & $P 2 / c$ \\
\hline & $2^{6}$ & 34 & $C 222$ \\
\hline & $2^{6}$ & 26 & $P 222$ \\
\hline & $2^{6}$ & 21 & $P 2 / c$ \\
\hline & $4^{4}$ & 17 & $P \overline{4}$ \\
\hline & $2^{8}$ & 4 & $P \overline{1}$ \\
\hline \multirow{22}{*}{ hat } & $22 \star 2$ & 116 & $P 4_{2} / n b c$ \\
\hline & $42 \star$ & 115 & $P 4_{2} / m b c$ \\
\hline & $42 \star$ & 113 & $P \overline{4} c 2$ \\
\hline & $2 \star 222$ & 112 & $P 4_{2} 22$ \\
\hline & $4 \star 22$ & 111 & $P 4_{2} / m$ \\
\hline & $22 \star 2$ & 109 & $P 4_{2} / n m c$ \\
\hline & $2 \star 44$ & 108 & $P 4_{2} / n m c$ \\
\hline & $2 \star 222$ & 107 & $P 4_{2} / m m c$ \\
\hline & $222 \star$ & 100 & $C 2 / m$ \\
\hline & $222 \star$ & 95 & $P 2 / c$ \\
\hline & $222 \star$ & 87 & $\mathrm{Cccm}$ \\
\hline & $22 \star 22$ & 86 & Ccca \\
\hline & $22 \star 22$ & 77 & $P 4_{2} 22$ \\
\hline & $2 \star 2^{4}$ & 72 & $P 2 / m$ \\
\hline & $22 \star 22$ & 68 & $P \overline{4} m 2$ \\
\hline & $22 \star 22$ & 65 & Pmmn \\
\hline & $44 \star$ & 64 & $P \overline{4}$ \\
\hline & $44 \star$ & 62 & $P 4_{2} / m$ \\
\hline & $222 \star$ & 60 & $C 222$ \\
\hline & $22 \star \star \star$ & 56 & $P 2$ \\
\hline & $2^{4} \star$ & 45 & $P 2_{1} / m$ \\
\hline & $2^{4} \star$ & 41 & $C 2 / c$ \\
\hline
\end{tabular}

modulo $\circ \circ \circ$ ), written in terms of the three reflections in the boundary edges of the $\star 246$ domain. These reflections, $r_{1}, r_{2}$ and $r_{3}$, are listed as the matrix operations describing their corresponding euclidean isometries in $\mathbb{E}^{3}$ in Table 9 for the $P$ and $D$ surfaces. The $\star 246$ reflections are not euclidean isometries on the Gyroid. Rather, pairs of reflections, corresponding to the three rotation group generators $\left(R_{1}, R_{2}\right.$ and $R_{3}$ ) of the stellate subgroup of $\star 246$, namely 246 , are used to generate space groups in Table 10 . We therefore generate space groups via the Gyroid from orbifolds containing rotations only (i.e. stellate and toroidal orbifolds) that lie within the lattice of orbifolds (described above) spanning $\star 246$ and $\circ \circ \circ$.

Given the correspondence between the set of group words mapped onto the $P, D$ and Gyroid TPMSs, we can generate 
Table 17

Correspondence between two-dimensional orbifolds and threedimensional euclidean space groups on the CLP surface: multiply connected orbifolds.

\begin{tabular}{|c|c|c|c|c|c|c|c|}
\hline \multirow{2}{*}{\multicolumn{4}{|c|}{$\begin{array}{l}\text { Correspondence between two-dimensional orbifolds and three- } \\
\text { dimensional euclidean space groups on the } C L P \text { surface: multiply }\end{array}$}} & \multirow{3}{*}{ Class } & \multirow[b]{2}{*}{ Orbifold } & \\
\hline & & & & & & Group No. & 3D space group \\
\hline connect & & & & & $\circ 2^{4}$ & 10 & $P 2$ \\
\hline Class & Orbifold & Group No. & 3D space group & & $\circ 2^{4}$ & 8 & $P 2$ \\
\hline & & & & & $\infty$ & 7 & $P c$ \\
\hline projective & $222 \times$ & 93 & $P \overline{4} 2 c$ & & $\circ \circ \circ$ & 1 & $P 1$ \\
\hline & $44 \times$ & 92 & $P \overline{4} 2_{1} c$ & & & & \\
\hline & $222 \times$ & 75 & $P 4_{2} 2_{1} 2$ & & & & \\
\hline & $222 \times$ & 73 & $P 4_{2} / n$ & & & & \\
\hline & $2^{4} \times$ & 22 & $P 1^{270}$ & Table 18 & & & \\
\hline & $2 \times x$ & 94 & $P \overline{4} 2_{1} c$ & Correspondence & between & mensional & Is and three- \\
\hline & $2 \times \times$ & 71 & $P 4_{2} b c$ & dimensional euclid & lean space & on the $H$ su & \\
\hline & $\begin{array}{l}x \times \times \\
22 \times x\end{array}$ & $\begin{array}{l}54 \\
53\end{array}$ & $\begin{array}{l}P c \\
C 2\end{array}$ & Class & Orbifold & Group No. & 3D space group \\
\hline & $22 \times x$ & 52 & $C 2 / c$ & & & & \\
\hline & $x \times x$ & 51 & $P 2_{1} / c$ & coxeter & $\star 2226$ & 32 & $\mathrm{~Pb}_{3} / \mathrm{mmc}$ \\
\hline & $22 \times x$ & 32 & $\mathrm{P4}_{2}$ & & $\star 2^{4} 3$ & 26 & $P \overline{6} m 2$ \\
\hline & $22 \times x$ & 31 & $C c c 2$ & & $\star 2266$ & 25 & $P \overline{3} m 1$ \\
\hline & $22 \times \times$ & 23 & $P 2_{1} 2_{1}$ & stellate & 2226 & 31 & $P \overline{3} 1 c$ \\
\hline & $22 \times \times$ & 18 & $P P^{\bar{L}_{1}{ }^{2}}$ & & 2266 & 23 & $P \overline{3}$ \\
\hline & $x^{4}$ & 16 & $C c$ & & $2^{4} 3$ & 22 & P312 \\
\hline & $x^{4}$ & 13 & $\begin{array}{l}C C \\
P 2_{1}\end{array}$ & & $2^{6}$ & 15 & $C 2 / c$ \\
\hline & $x^{4}$ & 6 & $\begin{array}{l}P Z_{1} \\
P 1\end{array}$ & & $2^{8}$ & 2 & $P \overline{1}$ \\
\hline & $x^{4}$ & 2 & $P \overline{1}$ & hat & $26 \star$ & 30 & $P 6_{3} / m$ \\
\hline möbius & $2 \star x$ & 89 & $\mathrm{P}_{2} / \mathrm{m}$ & & $22 \star 3$ & 29 & $P 6_{3} 22$ \\
\hline & $\star 22 \times$ & 88 & $P 4,2,2$ & & $2 \star 2^{4}$ & 24 & $\mathrm{Cmcm}$ \\
\hline & $2 \star x$ & 78 & $P_{4}$ & & $22 \star 2^{4}$ & 11 & $C 2 / m$ \\
\hline & $\star 22 x$ & 70 & $P 4_{2} m c$ & & $2^{4} \star$ & 10 & $P 2_{1} / m$ \\
\hline & $\star \times \times$ & 58 & $\begin{array}{l}F 4_{2} m c \\
A b a 2\end{array}$ & projective & $3 \times x$ & 21 & $P 6_{3} / m$ \\
\hline & $\star x \times$ & 37 & Pmn $2_{1}$ & & $x^{4}$ & 4 & $P 2_{1} / m$ \\
\hline & $\star \star x \times$ & 29 & $\begin{array}{l}P m n L_{1} \\
P 2 / c\end{array}$ & möbius & $22 \star x$ & 16 & $C 222_{1}$ \\
\hline & $\star \star x$ & 25 & $\mathrm{Pm}$ & & $\star 2^{4} \times$ & 14 & Amm2 \\
\hline annular & $\star \star 2$ & 114 & $P 4_{2} / m b c$ & & $\star \star \times$ & 13 & Ama2 \\
\hline & $\star \star 2$ & 105 & $\begin{array}{l}\mathrm{P}_{2} / \mathrm{mDC} \\
\mathrm{P}_{2} \mathrm{Cm}\end{array}$ & & $\star \star x$ & 12 & $\mathrm{Cmc2} 2_{1}$ \\
\hline & $\star \star 22$ & 102 & Amm 2 & & $\star \star x \times$ & 8 & $C 2$ \\
\hline & $\star \star 22$ & 101 & $\begin{array}{l}\text { Amm } \\
\text { Ccca }\end{array}$ & & $\star \star x \times$ & 5 & $\mathrm{Cm}$ \\
\hline & $2 \star \star$ & 99 & $\begin{array}{l}\text { Pcca } \\
\text { Pma2 }\end{array}$ & annular & $\star \star 3$ & 28 & $P \overline{6} 2 c$ \\
\hline & $2 \star \star$ & 96 & $\begin{array}{l}\text { Pmaz } \\
\text { Pmna }\end{array}$ & & $\star \star 3$ & 27 & $P 6_{3} m c$ \\
\hline & $22 \star \star$ & 85 & $\begin{array}{l}\text { Pmna } \\
\text { Cccm }\end{array}$ & & $\star \star 33$ & 19 & $P 3 m 1$ \\
\hline & $\star 2 \star 2$ & 81 & $\begin{array}{l}c c m \\
P \overline{4} b 2\end{array}$ & & $3 \star \star$ & 18 & $P \overline{6}$ \\
\hline & $2 \star \star$ & 76 & $P c c 2$ & & $\star 3 \star 3$ & 17 & P321 \\
\hline & $\star 2 \star 2$ & 69 & $\mathrm{P}_{2} \mathrm{mc}$ & & $0 \star \star$ & 3 & $\mathrm{Pm}$ \\
\hline & $2 \star \star$ & 66 & Pbam & toroidal & $\circ 3$ & 20 & $P \overline{6} 2 c$ \\
\hline & $\star \star 22$ & 61 & Cmm2 & & ○33 & 9 & $P 3$ \\
\hline & $\circ \star$ & 57 & $C 2 / c$ & & $\circ 2^{4}$ & 7 & $C 2$ \\
\hline & $22 \star \star$ & 56 & $\begin{array}{l}C 2 / C \\
P 2\end{array}$ & & ০০ & 6 & $C c$ \\
\hline & $22 \star \star$ & 49 & Ama2 & & ००० & 1 & $P 1$ \\
\hline
\end{tabular}

$\star \star \star$

$\star \star \star$

$22 \star \star$

$\star \star 2^{4}$

$\circ \star$

$\star \star \star$

$22 \star \star$

$\star \star \star \star$

$\star \star 2^{4}$

$22 \star \star$

$\star 22 \star 22$

$22 \star \star$

$\star 22 \star 22$

$\circ \star \star$

$0 \star \star$

$\star^{4}$

toroidal
Table 17 (continued)

Table 18 dimensional euclidean space groups on the $H$ surface.

the orbit of a point in $\mathbb{E}^{3}$ in general position. We can therefore determine the space group or each hyperbolic group-TPMS couple. We have used the PLATON software to extract these space groups numerically (Spek, 2003).

\section{A2. $C L P, H$ and $I-W P$ embeddings}

An analogous procedure to that used for the $P, D$ and Gyroid surfaces is used for the additional genus-three TPMSs, the $C L P$ and $H$ surfaces. In these cases, all subgroups of the full TPMS hyperbolic orbifolds, namely $\star 2224(C L P)$ and $\star 2226(H)$, are enumerated that are also supergroups of the $\circ \circ \circ$ translational subgroups of these surfaces. The procedure for generating these groups (and the group numbering we use here) for the $H$ surface is discussed in detail in a previous article (Robins et al., 2004b). Since both genus-three TPMSs have coxeter orbifolds with four corners, we generate the orbits of generic points in $\mathbb{E}^{3}$ via the Cartesian operators for the isometries in $\mathbb{E}^{3}$ corresponding to two-dimensional 
Table 19

Subgroups of $2 \star 24$ commensurate with a primitive translational unit cell of the $I-W P$ surface.

\begin{tabular}{|c|c|c|c|c|}
\hline & Orbifold & Index & $\begin{array}{l}\text { Conjugacy } \\
\text { class size }\end{array}$ & $\begin{array}{l}\text { Subgroup } \\
\text { generators in } 2 \star 24 / T\end{array}$ \\
\hline 1 & $\circ \circ \circ \circ$ & 48 & 1 & identity \\
\hline 2 & $\times \times \times \times \times$ & 24 & 1 & $r_{1} R_{1} r_{1} R_{1} r_{1} R_{1}$ \\
\hline 3 & $\circ \circ 22$ & 24 & 3 & $r_{2} R_{1} r_{2} R_{1}$ \\
\hline 4 & $\star \times \times \times \times$ & 24 & 3 & $r_{2}$ \\
\hline 5 & $\circ 222222$ & 24 & 6 & $r_{1} r_{2}$ \\
\hline 6 & $\star \times \times \times \times$ & 24 & 6 & $r_{1}$ \\
\hline 7 & ০ & 16 & 4 & $r_{1} r_{2} R_{1}$ \\
\hline 8 & $\circ 222$ & 12 & 1 & $R_{1} r_{2} R_{1}^{-1} r_{2}^{-1}, r_{1} R_{1} r_{2} R_{1}^{-1} r_{2}^{-1} r_{1}^{-1}$ \\
\hline 9 & $2 \star \times \times$ & 12 & 3 & $r_{2}, R_{1} r_{1} R_{1} r_{1}^{-1} R_{1}^{-1} r_{1}^{-1}$ \\
\hline 10 & 2222222 & 12 & 3 & $r_{2} r_{1}^{-1}, R_{1} r_{1} R_{1} r_{1}^{-1} R_{1}^{-1}$ \\
\hline 11 & $\star 22 \times \times$ & 12 & 3 & $r_{1}, R_{1} r_{1} R_{1} r_{1}^{-1} R_{1}^{-1} r_{2}^{-1}$ \\
\hline 12 & $2 \times \times \times$ & 12 & 3 & $R_{1} r_{2}^{-1}$ \\
\hline 13 & $\circ 44$ & 12 & 3 & $R_{1} r_{2} R_{1}^{-1} r_{2}^{-1}, r_{1} R_{1} r_{1} R_{1}^{-1} r_{2}^{-1} r_{1}^{-1}$ \\
\hline 14 & $\circ \star 22$ & 12 & 3 & $r_{2}, R_{1} r_{2} R_{1}^{-1}$ \\
\hline 15 & $22 \star 22 \times$ & 12 & 6 & $r_{1}, r_{2}$ \\
\hline 16 & $222 \star \times$ & 12 & 6 & $r_{1}, R_{1} r_{1} R_{1} r_{1}^{-1} R_{1}^{-1}$ \\
\hline 17 & $\times \times \times$ & 8 & 4 & $r_{1} r_{2} R_{1}^{-1}, r_{1} R_{1} r_{1} R_{1}^{-1} r_{2}^{-1}$ \\
\hline 18 & 222222 & 8 & 4 & $r_{2} r_{1}^{-1}, R_{1}$ \\
\hline 19 & $\circ \star$ & 8 & 4 & $r_{1} r_{2} R_{1}^{-1}, r_{1} R_{1} r_{1} R_{1}^{-1} r_{1}^{-1}$ \\
\hline 20 & $\star 222 \times$ & 6 & 1 & $r_{2}, R_{1} r_{2} R_{1}^{-1}, r_{1} R_{1} r_{2} R_{1}^{-1} r_{1}^{-1}$ \\
\hline 21 & $\star 44 \times$ & 6 & 3 & $r_{1}, R_{1} r_{2} R_{1}^{-1}$ \\
\hline 22 & $2 \star 2 \times$ & 6 & 3 & $r_{1}, R_{1} r_{2} R_{1}^{-1} r_{2}^{-1}$ \\
\hline 23 & $222 \star 2$ & 6 & 3 & $r_{2}, R_{1}$ \\
\hline 24 & $22 \star 222$ & 6 & 3 & $r_{1}, r_{2}, R_{1} r_{1} R_{1} r_{1}^{-1} R_{1}^{-1}$ \\
\hline 25 & $4 \star \times$ & 6 & 3 & $r_{2}, R_{1} r_{2} R_{1}^{-1} r_{1}^{-1}$ \\
\hline 26 & $2^{4} 4$ & 6 & 3 & $r_{2} r_{1}^{-1}, R_{1} r_{2} R_{1}^{-1} r_{1}^{-1}$ \\
\hline 27 & $\circ 2$ & 4 & 1 & $r_{1} r_{2} R_{1}^{-1}, r_{1} R_{1} r_{2}^{-1}$ \\
\hline 28 & $222 \star$ & 4 & 4 & $r_{2} r_{1}^{-1}, R_{1}, r_{1} R_{1} r_{1} R_{1}^{-1} r_{1}^{-1}$ \\
\hline 29 & $2 \star 224$ & 3 & 3 & $r_{1}, r_{2}, R_{1} r_{2} R_{1}^{-1}$ \\
\hline 30 & $\star 2 \times$ & 2 & 1 & $r_{2}, R_{1} r_{1}^{-1}$ \\
\hline 31 & $\star 2 \times$ & 2 & 1 & $r_{1}, R_{1} r_{2}^{-1}$ \\
\hline 32 & 2224 & 2 & 1 & $r_{2} r_{1}^{-1}, R_{1}, r_{1} R_{1} r_{1}^{-1}$ \\
\hline 33 & $2 \star 24$ & 1 & 1 & $r_{1}, r_{2}, R_{1}$ \\
\hline
\end{tabular}

reflections in the four boundary edges of their orbifolds $\left(r_{1}, r_{2}\right.$, $r_{3}$ and $r_{4}$ ). Those hyperbolic reflections induce twofold rotations $\left(r_{1}\right)$ and three-dimensional reflections $\left(r_{2}, r_{3}, r_{4}\right)$ in the $H$ surface, respectively (see Robins et al., 2004b). Those isometries are listed in Table 13. These generate space groups via the process described in the previous section.

The hyperbolic group presentation for the CLP surface has not previously been published so we give details here. The $\star 2224$ group is generated by reflections, $r_{1}, r_{2}, r_{3}$ and $r_{4}$, in the four sides of the quadrilateral so that rotations $r_{1} r_{2}, r_{2} r_{3}$ and $r_{4} r_{1}$ are of order 2 and $r_{3} r_{4}$ is of order 4 . The genus- 3 translational subgroup $T$, with orbifold $\circ \circ \circ$ is then defined by the following translations:

$$
\begin{aligned}
t_{a} & =r_{4} r_{2} r_{4} r_{2}, \\
t_{b} & =r_{3} r_{1} r_{3} r_{1}, \\
t_{c} & =r_{4} r_{3} r_{1} r_{3} r_{1} r_{4}, \\
t_{d} & =r_{4} r_{3} r_{4} r_{2} r_{4} r_{2} r_{3} r_{4}, \\
t_{e} & =r_{4} r_{3} r_{4} r_{3} r_{1} r_{3} r_{1} r_{4} r_{3} r_{4}, \\
t_{f} & =r_{3} r_{4} r_{3} r_{2} r_{4} r_{2} r_{4} r_{3} r_{4} r_{3}, \\
t_{g} & =r_{3} r_{4} r_{1} r_{3} r_{1} r_{3} r_{4} r_{3}, \\
t_{h} & =r_{3} r_{2} r_{4} r_{2} r_{4} r_{3}
\end{aligned}
$$

These details are illustrated in Fig. 8.

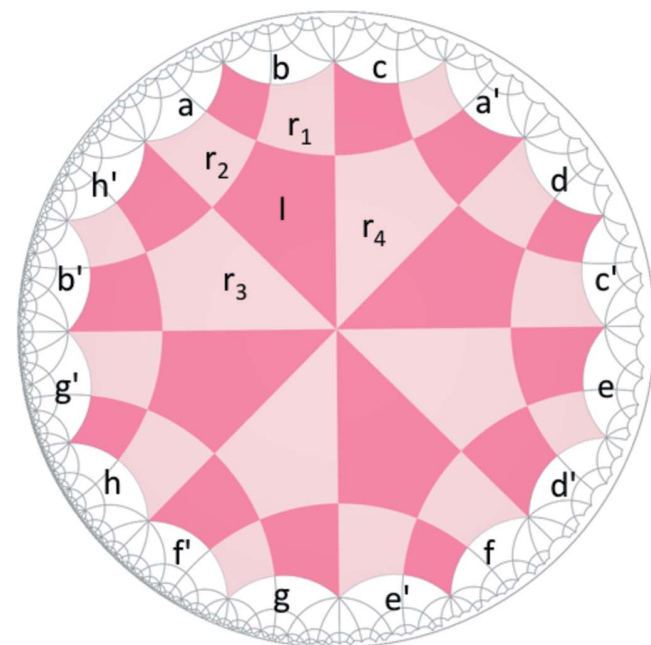

(a)

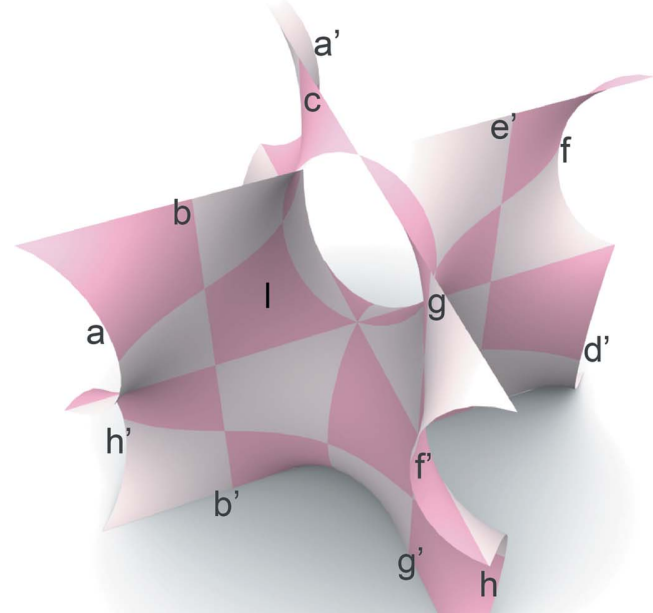

Figure 8

(b)

Hyperbolic geometry of the $C L P$ surface. (a) A fundamental region for the $\star 2224$ symmetry group is marked $I$, and the images of $I$ under reflection in its four sides are labelled by the appropriate reflection. The 32 coloured quadrilateral domains define a translational unit cell for the $\circ \circ \circ$ domain of the $C L P$ surface. The translations $t_{a}, \ldots, t_{h}$ defined in the text are such that they map the edge marked $a$, say, onto the edge marked $a^{\prime}$. (b) A translational unit cell of the CLP surface corresponding to the illustrated hyperbolic domain.

The index of $\star 2224$ in $\circ \circ \circ$ is 32 ; as explained above this is the number of quadrilateral fundamental domains that make a translational unit cell. There are 118 subgroups that are simultaneously subgroups of $\star 2224$ and supergroups of $\circ \circ \circ$. Generators for these subgroups are provided in Table 15.

The $I$-WP surface is the only genus-four TPMS known. We enumerate all two-dimensional groups that lie between the full symmetry of this surface, namely $2 \star 24$, and its translational subgroup, $\circ^{4}$. The hat orbifold $2 \star 24$ is defined by three generators: $R_{1}$ representing the 2 rotation centre, and two to describe the pair of reflections, $r_{1}, r_{2}$, with $r_{1} r_{2}$ being an order-2 rotation, while the order- 4 rotations are given by $R_{1} r_{1} R_{1} r_{2}$, and 


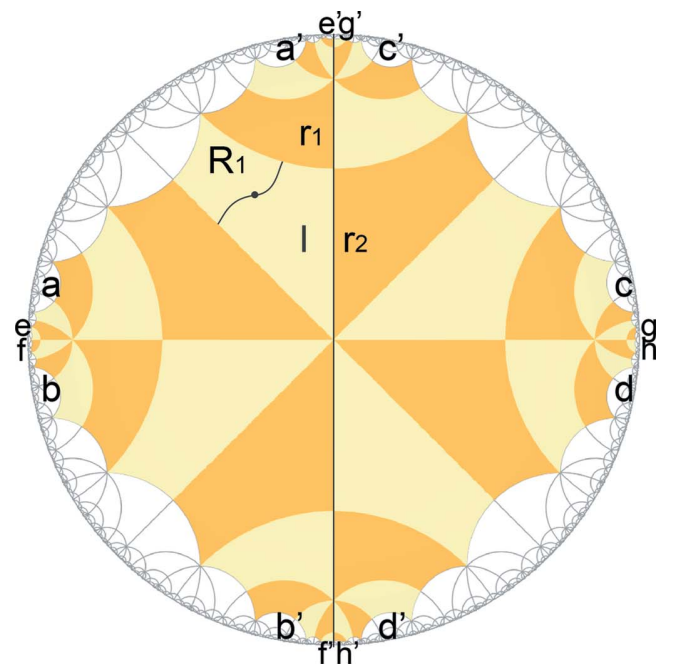

(a)

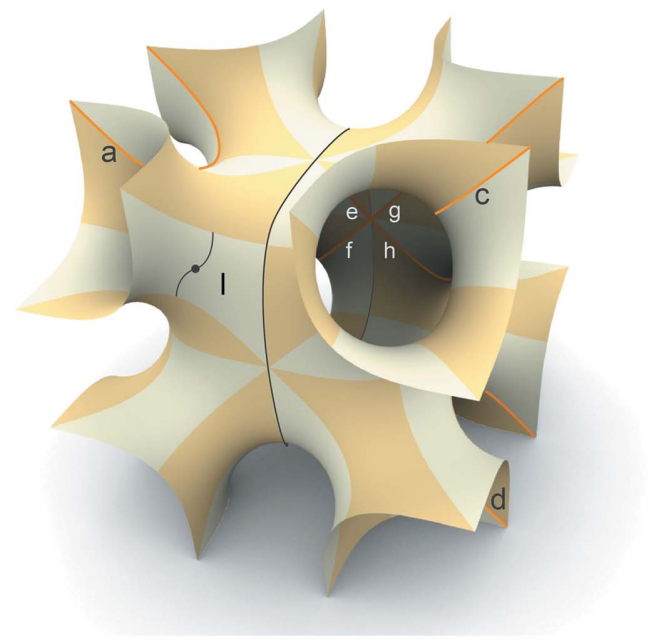

(b)

Figure 9

(a) Hyperbolic geometry of the $I-W P$ surface. A fundamental region for the $2 \star 24$ symmetry group is marked $I$, and the images of $I$ under rotation and reflection generators are labelled by the appropriate symbol. The 48 coloured quadrilaterals are doubled fundamental regions bounded by mirror lines and they cover the full body-centred translational cell, shown in $(b)$. (b) A primitive unit cell for the surface is found by cutting this region in half. The black line in $\mathbb{H}^{2}$ maps to a vertical plane in $\mathbb{E}^{3}$, as shown. The 'cut lines' marked by letters $a-h$ correspond to the paired labels in the hyperbolic domain.

$R_{1} r_{2} R_{1} r_{1}$. These generators (illustrated in Fig. 9) are converted to isometries of $\mathbb{E}^{3}$, listed in Table 14 .

The genus-4 translational subgroup is defined by the following hyperbolic translations: (the $g_{i}$ refer to translations that glue-up within a unit cell of the surface while the $t_{j}$ map to translations in $\mathbb{E}^{3}$.)

$$
\begin{aligned}
& g_{1}=\left(R_{1} r_{1}\right)^{6}, \\
& g_{2}=\left(R_{1} r_{2}\right)^{4}, \\
& g_{3}=r_{1}\left(R_{1} r_{2}\right)^{4} r_{1}, \\
& g_{4}=R_{1} r_{1}\left(R_{1} r_{2}\right)^{4} r_{1} R_{1}, \\
& g_{5}=r_{1} R_{1} r_{1}\left(R_{1} r_{2}\right)^{4} r_{1} R_{1} r_{1},
\end{aligned}
$$

\begin{tabular}{|c|c|c|c|}
\hline Class & Orbifold & Group No. & 3D space group \\
\hline coxeter & - & & \\
\hline \multirow[t]{4}{*}{ stellate } & 2224 & 32 & I 432 \\
\hline & $2^{4} 4$ & 26 & I 422 \\
\hline & $2^{6}$ & 18 & $R 32$ \\
\hline & $2^{7}$ & 10 & $F 222$ \\
\hline \multirow[t]{5}{*}{ hat } & $2 \star 24$ & 33 & $\operatorname{Im} \overline{3} m$ \\
\hline & $2 \star 224$ & 29 & $I 4 / \mathrm{mmm}$ \\
\hline & $222 \star$ & 28 & $R^{\frac{1}{3}} m$ \\
\hline & $22 \star 222$ & 24 & Fmmm \\
\hline & $222 \star 2$ & 23 & $I \overline{4} m 2$ \\
\hline \multirow[t]{3}{*}{ projective } & $x \times x$ & 17 & $R \overline{3}$ \\
\hline & $2 \times x \times$ & 12 & $I \overline{4}$ \\
\hline & $x^{5}$ & 2 & $P \overline{1}$ \\
\hline \multirow[t]{12}{*}{ möbius } & $\star 2 x$ & 31 & $I \overline{4} 3 m$ \\
\hline & $\star 2 x$ & 30 & $\operatorname{Im} \overline{3}$ \\
\hline & $4 \star x$ & 25 & $I 4 / m$ \\
\hline & $2 \star 2 x$ & 22 & $I \overline{4} 2 m$ \\
\hline & $\star 44 \times$ & 21 & $I 4 \mathrm{~mm}$ \\
\hline & $\star 222 x$ & 20 & Immm \\
\hline & $222 \star x$ & 16 & $C 2 / m$ \\
\hline & $22 \star 22 x$ & 15 & $F m m 2$ \\
\hline & $\star 22 \times \times$ & 11 & Fmm 2 \\
\hline & $2 \star x \times$ & 9 & $C 2 / m$ \\
\hline & $\star x^{4}$ & 6 & $\mathrm{Cm}$ \\
\hline & $\star x^{4}$ & 4 & $\mathrm{Cm}$ \\
\hline \multirow[t]{2}{*}{ annular } & $\circ \star$ & 19 & $R 3 m$ \\
\hline & $\circ \star 22$ & 14 & $\operatorname{Imm} 2$ \\
\hline \multirow[t]{7}{*}{ toroidal } & $\circ 2$ & 27 & $I 23$ \\
\hline & $\circ 44$ & 13 & I4 \\
\hline & o222 & 8 & $I 222$ \\
\hline & ০o & 7 & $R 3$ \\
\hline & $\circ 2^{6}$ & 5 & $C 2$ \\
\hline & $\circ \circ 22$ & 3 & $C 2$ \\
\hline & $0^{4}$ & 1 & $P 1$ \\
\hline
\end{tabular}

Table 20

Two-dimensional-three-dimensional symmetries on the $I-W P$ surface.

$$
\begin{aligned}
g_{6} & =\left(R_{1} r_{1}\right)^{2}\left(R_{1} r_{2}\right)^{4}\left(r_{1} R_{1}\right)^{2}, \\
g_{7} & =r_{1}\left(R_{1} r_{1}\right)^{2}\left(R_{1} r_{2}\right)^{4}\left(r_{1} R_{1}\right)^{2} r_{1}, \\
t_{1} & =R_{1} r_{2} R_{1} r_{1} R_{1} r_{2} R_{1} r_{2} R_{1} r_{1} R_{1} r_{1} R_{1}, \\
t_{2} & =R_{1} r_{1} R_{1} r_{1} r_{2} R_{1} r_{1} R_{1} r_{2} R_{1} r_{2}, \\
t_{3} & =r_{1} R_{1} r_{2} R_{1} r_{2} R_{1} r_{1} R_{1} r_{1} r_{2} R_{1}, \\
t_{4} & =R_{1} r_{1} R_{1} r_{2} R_{1} r_{2} R_{1} r_{1} R_{1} r_{1} r_{2}, \\
t_{5} & =r_{1} R_{1} r_{1} r_{2} R_{1} r_{1} R_{1} r_{2} R_{1} r_{2} R_{1}, \\
t_{6} & =R_{1} r_{1} R_{1} r_{1} R_{1} r_{1} r_{2} R_{1} r_{1} R_{1} r_{2} R_{1} r_{1} r_{2} R_{1}, \\
t_{7} & =r_{1} R_{1} r_{1} R_{1} r_{2} R_{1} r_{2} R_{1} r_{1} R_{1} r_{2}, \\
t_{8} & =R_{1} r_{1} R_{1} r_{1} R_{1} r_{1} r_{2} R_{1} r_{1} r_{2} R_{1} r_{2} R_{1} r_{1} R_{1} .
\end{aligned}
$$

The index of $2 \star 24$ in $\circ \circ \circ$ is 48 ; the number of quadrilateral fundamental domains that make a translational unit cell. There are 33 subgroups that are subgroups of $2 \star 24$ and supergroups of $\circ^{4}$. Generators for these subgroups are provided in Table 19.

\section{References}

Atractor (2010). Simetria. http://www.atractor.pt/publicacoes/ritmo. htm.

Baez, J. (accessed 2013). Klein's quartic curve. http://math.ucr.edu/ home/baez/klein.html.

Bernal, J. (1966). Principles of Biomolecular Organization, Introduction. Boston: Little Brown and Co. 
Conway, J. \& Huson, D. (2002). Struct. Chem. 13, 247-256.

Conway, J. H. (1992). Groups, Combinatorics and Geometry, pp. 438447. Cambridge University Press.

Conway, J. H., Burgiel, H. \& Goodman-Strauss, C. (2008). Editors. The Symmetries of Things. Wellesley: AK Peters Ltd.

Conway, J. H., Friedrichs, O. D., Huson, D. H. \& Thurston, W. P. (2001). Beitr. Algebra Geom. 42, 475-507.

Coxeter, H. (1934). Ann. Math. 35, 588-621.

Fogden, A. \& Hyde, S. T. (1992). Acta Cryst. A48, 575-591.

Hahn, Th. (1992). International Tables of Crystallography, Vol. A, 3rd ed. Dordrecht: Kluwer.

Huson, D. (accessed 2013). Two-dimensional symmetry mutation. http://citeseerx.ist.psu.edu/viewdoc/download?doi=10.1.1.30.8536 $\&$ rep $=$ rep1\&type $=$ pdf.

Hyde, S., Andersson, S., Blum, Z., Lidin, S., Larsson, K., Landh, T. \& Ninham, B. (1997). The Language of Shape. Amsterdam: Elsevier Science BV.

Hyde, S. \& Ramsden, S. (2003). Eur. Phys. J. 31, 273-284.

Hyde, S. T., Robins, V. \& Ramsden, S. J., (2010). Epinet. http:// epinet.anu.edu.au.

Johnson, C. K. \& Burnett, M. N., (1996). Crystallographic topology. http://web.ornl.gov/sci/ortep/topology/overview.html.

Kopsky, V. \& Litvin, D. B. (2010). Editors. International Tables for Crystallography, Vol. E. Chichester: Wiley.

Lenosky, T., Gonze, X., Teter, M. \& Elser, V. (1992). Nature (London), 355, 333-335.
Levy, S. (2001). The Eightfold Way: the Beauty of Klein's Quartic Curve. Cambridge: Cambridge University Press/MSRI Publications.

Lijima, S., Ichihashi, T. \& Ando, Y. (1992). Nature (London), 356, 776-778.

Mackay, A. L. (1986). Acta Cryst. A42, 55-56.

Molnar, E. (2002). Struct. Chem. 13, 267-275.

Montesinos, J. M. (1987). Classical Tessellations and Three-Manifolds, p. VIII. Berlin: Springer-Verlag.

O'Keeffe, M., Peskov, M. A., Ramsden, S. J. \& Yaghi, O. M. (2008). Acc. Chem. Res. A, 41, 1782-1789.

Ramsden, S. J., Robins, V. \& Hyde, S. T. (2009). Acta Cryst. A65, 81108.

Ratcliffe, J. G. (1994). Foundations of Hyperbolic Manifolds. New York: Springer Verlag.

Robins, V., Hyde, S. \& Ramsden, S. (2004a). Eur. Phys. J. B, 39, $365-$ 375.

Robins, V., Hyde, S. \& Ramsden, S. (2004b). Physica A, 339, 173-180.

Robins, V., Ramsden, S. \& Hyde, S. (2005). Eur. Phys. J. B, 48, 107111.

Shevchenko, V. Ya., Madison, A. E. \& Mackay, A. L. (2007). Acta Cryst. A63, 172-176.

Spek, A. L. (2003). J. Appl. Cryst. 36, 7-13.

Stillwell, J. (1980). Classical Topology and Combinatorial Group Theory. New York: Springer.

Stillwell, J. (1992). Geometry of Surfaces. New York: Springer-Verlag. 